


\section{GATT Standards Code Activities of the National Institute of Standards and Technology - 1990}

\section{JoAnne R. Overman}

U.S. DEPARTMENT OF COMMERCE National Institute of Standards and Technology

Standards Code and Information Program Office of Standards Services

Gaithersburg, MD 20899

April 1991

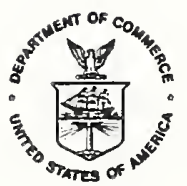





\section{ABSTRACT}

This report describes the GATT standards code activities conducted by the Standards Code and Information Program, National Institute of standards and Technology (NIST), for calendar year 1990. NIST responsibilities include operating the U.S. GATT inquiry point for information on standards and certification activities and the technical office for non-agricultural products; notifying the GATT Secretariat of proposed U.S. Federal Government standards-based rules that might significantly affect trade; assisting U.S. industry with standards-related trade problems; and responding to inquiries about proposed foreign and U.S. regulations.

Key Words: certification information; GATT Standards code; notifications; proposed foreign regulations; standards information; technical assistance 
CONTENTS

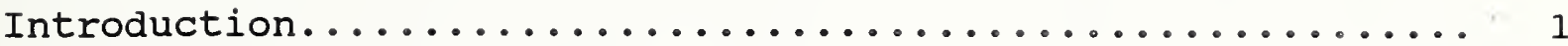

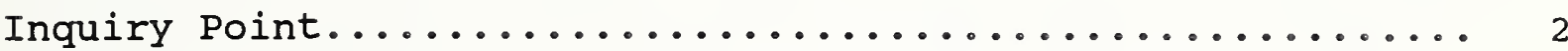

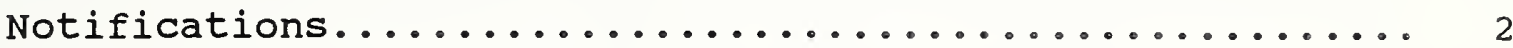

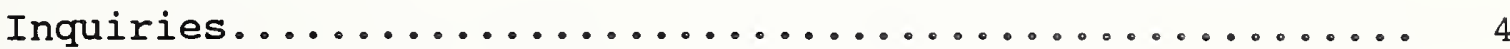

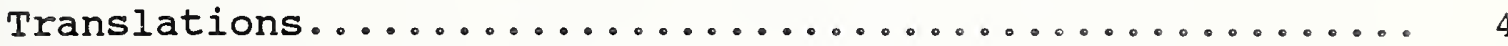

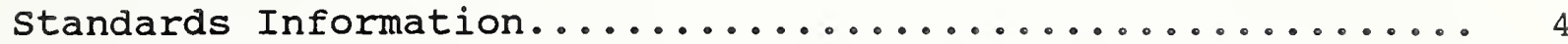

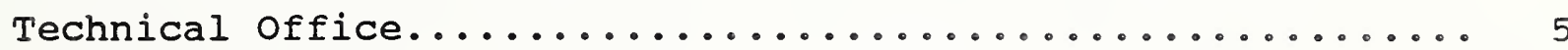

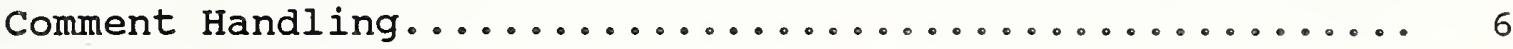

Comment Periods............................. 6

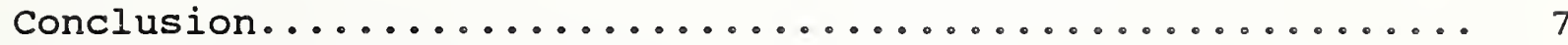

Standards Code signatories..................... 8

Tables

Table I Number of Notifications Issued in $1990 \ldots . . .99$

Table II List of U.S. Notifications............... 10

Table III List of Foreign Notifications............. 11

Appendix A Key-Word-Out-of-Context (KWOC) Index of

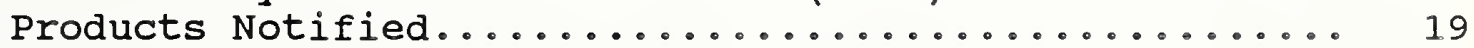

Appendix B Number of Enquiries by Signatories ........ 47 



\section{INTRODUCTION}

The Agreement on Technical Barriers to Trade (popularly known as the Standards Code) was negotiated and accepted during the Tokyo Round of Multilateral Trade Negotiations, which took place between 1975 and 1979 under the auspices of the General Agreement on Tariffs and Trade (GATT). The standards Code is designed to eliminate the use of standards, technical regulations and conformity assessment procedures as unnecessary barriers to trade.

There are currently 40 signatories to the Standards Code; Israel signed in 1990. Thirty of the signatories have actively implemented the code in their countries. The other 10 signatories have either not: 1) ratified the Agreement; 2) established an inquiry point for standards information; 3) reported any proposed government mandatory regulations (see "Standards Code Signatories"); or 4) some combination of the foregoing. The code is administered by the GATT secretariat in Geneva, Switzerland.

In 1990, the new round of multilateral trade negotiations - known as the Uruguay Round - was officially completed. Due to conflicts in non-standards related areas, the negotiations were not finished and new proposals to the standards code were not adopted. As this report goes to press, negotiations have been resumed.

The Trade Agreements Act of 1979 ratified the United States' accession to the GATT Standards code. Under Title IV of the Act, the NIST Office of Standards services (OSS) is responsible for several provisions which relate to the establishment of a U.S. inquiry point, 1 a standards information center, and a technical office for non-agricultural products.

This report summarizes standards code activities conducted by the Standards code and Information (SCI) Program for calendar year 1990. These functions are performed within the traditional framework of NIST's standards-related programs.

1 The Standards code requires that each signatory provide an inquiry point to answer all requests for information about technical regulations, standards, and rules of conformity assessment. 


\section{Notifications}

Signatories of the standards code must notify the GATT secretariat of all proposed government regulations and certification procedures that might significantly affect international trade. The secretariat, in turn, disseminates the notifications to all other signatories. As the official U.S. GATT inquiry point for information on standards and certification activities, SCI notifies the Secretariat of proposed U.S. Federal Government rules that might significantly affect trade. SCI also receives foreign notifications through the GATT secretariat and disseminates them, along with supplementary information, to appropriate U.S. companies and industry groups. SCI responds to inquiries about foreign and U.S. notifications, provides copies of the proposed regulations, and arranges for translations of foreign standards. SCI also assists in developing comments on the impact on U.S. industry of proposed foreign regulations and transmits them to the foreign government. concerned.

In 1990 the GATT Secretariat issued 362 notifications. Twentyfive signatories reported at least one proposed regulation. (See Table I.) Czechoslovakia had the most notifications (47). Twelve other signatories - Austria, Belgium, Denmark, EEC, Finland, Japan, Netherlands, New Zealand, Philippines, Sweden, United States, and Yugoslavia - each reported 10 or more proposed regulations.

An official in the office of the U.S. Trade Representative (USTR), based in Geneva, receives notifications from the GATT secretariat and cables them to the United States. The notifications, all of which are available in English, include the name of the country proposing the regulation; the assigned TBT/Notification number; a brief description of the regulation; and the final date for comments. In order to provide wide access to this information in the United states, all foreign notifications are published in the Commerce Business Daily and several other U.S. publications. SCI maintains a computerized database on all GATT notifications and a telephone GATT hotline where up-to-date notification information can be obtained. The hotline number is (301) 975-4041 (not toll free).

Proposed U.S. Regulations

All proposed U.S. Government rules (mandatory standards or conformity assessment systems), including proposed revisions, are published in the Federal Register by the responsible Federal agency. SCI staff regularly review the Register to identify those proposed regulations that should be notified to the GATT 
Secretariat and send notices directly to the GATT Secretariat via telefax.

In 1990, 30 proposed U.S. regulations, covering such products as motor vehicles, appliances, and foods, were reported. The originating agencies are shown below. (See table II for listing of specific products by agency.)

\section{Agency}

No. of 1990 Notifs.

Agriculture, Dept. of (USDA) Consumer Product Safety Commission (CPSC) Energy, Dept. of (DOE) Environmental Protection Agency (EPA) Federal Communications Commission (FCC) Food and Drug Administration (FDA) Housing \& Urban Development, Dept. of (HUD) 1 National Highway Traffic Safety Administration (NHTSA) Transportation, Dept. of (DOT)

7
Total $\quad \begin{array}{r}2 \\ \hline 30\end{array}$

Proposed Foreign Regulations

The GATT Secretariat in 1990 issued 332 notifications from countries other than the United States. (See table III.) The foreign regulations covered a wide variety of products, including bed warmers, fish farming, dentifrice, cooked ham, and mud additives. SCI maintains a Key-Word-Out-of-Context (KWOC) index for all products that are the subject of foreign notifications. (See Appendix A for the 1990 index.) (KWOC is an indexing method in which keywords, in this instance product names, appear as headings on a separate line.) The KWOC index is used to identify regulations that may be of interest to individuals who have expressed interest in specific products.

SCI regularly sends foreign notifications to members of the Industry Functional Advisory Committee on Standards (IFAC) ${ }^{2}$ and several other groups and specific individuals to serve their information needs and to allow them to disseminate this information to others. SCI sends copies of all foreign notifications to the American National Standards Institute for subsequent publication in its biweekly ANSI Reporter.

2 The IFAC is comprised of Government-appointed individuals from private industry engaged in standards-related activities. It advises the Department of Commerce and USTR on trade matters, the operation of any adopted trade agreements and other subjects related to U.S. trade policy. 
SCI responded to 407 domestic and foreign letter and telephone requests for GATT notification information (308 U.S., 99 foreign) in 1990. Many requestors asked for copies of more than one regulation. In addition to requests for specific regulations, many individuals asked for general information on the standards Code. SCI responds to such requests by sending an information package consisting of the latest issue of its newsletter, tbt news, a list of recent foreign notifications, brochures describing the standards code, and ordering information for the Commerce Business Daily.

In 1990, the most often requested regulations concerned telecommunications equipment, chemicals, ozone layer depleting substances (CFCs), metric use, medical products and electrical equipment. In addition, 392 individuals called the GATT hotline for up-to-date information on foreign notifications.

\section{Translations}

SCI requests the text of all proposed foreign regulations which are notified. Signatory countries are required to supply copies of the regulations through their inquiry points. Of the 319 regulations received in 1990, 189 were in languages other than English. SCI coordinates a service to obtain an English translation on request, of any proposed regulation in a foreign language. The cost of translation is paid by the requestor; if more than one request is received for the same translation, the cost is divided equally among requestors. In 1990, SCI received five requests for translation of five regulations and standardsrelated documents.

\section{STANDARDS INFORMATION}

The National Center for Standards and Certification Information (NCSCI) is the national focal point for standards-related information. NCSCI supports GATT inquiry point activities by supplying information concerning both domestic and foreign standards. The information center, established in 1965, provides government, industry and the public with information on standards, regulations, certification programs, and related activities that affect trade and commerce.

NCSCI maintains a microform and/or hard copy collection of engineering standards and specifications, test methods, regulations, certification rules, directories, reference books and special publications. In 1990, NCSCI staff responded to more than 5,000 requests from all over the world on the existence, source, and availability of standards and related documents. Thirty-two of the 40 signatories contacted the center for information. (See Appendix B.) NCSCI also responds to 
trade-related queries regarding regulations or other requirements imposed by foreign countries that affect the export of U.S. manufactured products.

In 1990, SCI published and NCSCI distributed the Directory of European Regional standards-Related Organizations (NIST SP 795) which lists European organizations involved in standards-related activities.

In a related area - the EC 1992 Single Internal Market - NCSCI established an EC hotline to report on draft CEN and CENELEC standards and EC directives related to standards which may affect trade. The recorded hotline - (301) 921-4164 (not toll-free) is updated weekly and provides the subject/product areas, document number and final date for comments. The hotline was established in August 1990 and over 800 calls have been received as of December 31, 1990.

NCSCI staff also review the official Journal of the European Communities to identify proposed standards-related directives. Copies of the directives are sent to the appropriate trade or professional associations for dissemination to and review by their members.

\section{TECHNICAL OFFICE}

The Technical office, established by the Trade Agreements Act of 1979, is responsible for providing technical support related to non-agricultural products. Office functions include:

a) participation in standards-related conferences; b) technical assistance to the International Trade Administration of the Department of Commerce and the office of the U.S. Trade Representative in developing proposals to enhance the standards Code; c) preparation of special reports on standards issues; and d) coordination of comments on proposed foreign regulations.

\section{Technical office activities included:}

- Technical office staff assisted the American Institute of Aeronautics and Astronautics in reviewing draft standards developed within their recently established standards program.

- Office staff participated in continuing bilateral discussions with Mexico standards officials to establish a Free Trade Agreement.

- Staff members continued their work with other Government agencies to inform U.S. industry of EC 1992 standards-related activities. These activities included participating in the EC 1992 Work Group on Standards, Testing and Certification and making presentations on EC 1992 standards development activities to interested U.S. industry groups. 
A major objective of the standards Code is to allow exporters, government agencies, and other interested parties to comment on proposed foreign regulations which they believe would unjustifiably impede exports. SCI provides the text of proposed foreign regulations on request and encourages the submission of comments if a regulation appears to create unnecessary trade barriers. SCI transmits comments to the appropriate foreign government and coordinates any follow-up activity. In 1990, SCI received and processed formal comments on four foreign regulations: Sweden - cosmetics (90.72) and emission parts for light vehicles $(90.237)$; EEC - pneumatic tires $(90.237)$; and Japan - cosmetics $(90.291)$.

Additional informal comments are frequently made by industry representatives who become aware of proposed regulations, often even before they are officially notified through the GATT. The notification system can often serve as a back-up mechanism to ensure awareness of foreign developments.

Sweden submitted comments on a proposed Food and Drug Administration proposal. The comments were forwarded to the appropriate FDA office for action.

\section{Comment periods}

So that signatories will have adequate time to receive the text of a regulation (and to have it translated if necessary), review it, and prepare comments, the standards code recommends a comment period of at least 60 days. If the comment period is insufficient (e.g., due to late receipt of an English version or complicated technical issues), the SCI staff will request that the issuing country extend the comment period. In 1990, eight such requests were made by the United states to the notifying parties. Four of the extension requests were granted. Many extensions are requested so that individuals can translate and study the regulation without necessarily indicating an intention to comment. SCI staff also received five requests from other signatories requesting extensions of the comment period for proposed U.S. technical regulations.

The average length of the comment period ${ }^{3}$ in 1990 was 49 days for U.S. regulations and 44 days for foreign regulations. The average length of the comment period, by U.S. agency, is shown on the following page.

3 Number of calendar days between the date the notification was issued by the GATT secretariat and the closing date for comments. 


$\begin{array}{ll}\text { CPSC } & 42 \text { days } \\ \text { DOE } & 62 \text { days } \\ \text { DOT } & 70 \text { days } \\ \text { EPA } & 64 \text { days } \\ \text { FCC } & 36 \text { days } \\ \text { FDA } & 62 \text { days } \\ \text { HUD } & 52 \text { days } \\ \text { NHTSA } & 32 \text { days } \\ \text { USDA } & 34 \text { days }\end{array}$

Comment periods for foreign regulations ranged from none at all to 407 days. The average length of comment period by country was:

$\begin{array}{lrlr}\text { Austria } & 72 \text { days } & \text { India } & 0 \text { days } \\ \text { Belgium } & 0 \text { days } & \text { Japan } & 56 \text { days } \\ \text { Brazil } & 0 \text { days } & \text { Korea } & 19 \text { days } \\ \text { Canada } & 25 \text { days } & \text { Netherlands } & 46 \text { days } \\ \text { Czechoslovakia } & 39 \text { days } & \text { New Zealand } & 36 \text { days } \\ \text { Denmark } & 30 \text { days } & \text { Norway } & 70 \text { days } \\ \text { EEC } & 39 \text { days } & \text { Philippines } & 1 \text { days } \\ \text { Finland } & 73 \text { days } & \text { Singapore } & 0 \text { days } \\ \text { France } & 0 \text { days } & \text { Sweden } & 60 \text { days } \\ \text { Germany } & 0 \text { days } & \text { Switzerland } & 71 \text { days } \\ \text { Hong Kong } & 43 \text { days } & \text { United Kingdom } & 17 \text { days } \\ \text { Hungary } & 0 \text { days } & \text { Yugoslavia } & 48 \text { days } \\ & & \end{array}$

SCI continues to work with U.S. industry to address standardsrelated trade problems and to assure wide distribution of relevant notifications of proposed foreign regulations which might affect trade. Requests for further information, suggestions on how the service might be made more useful, or comments on subjects covered in this report should be directed to:

Standards code and Information Program National Institute of Standards and Technology Administration Building, Room A629

Gaithersburg, MD 20899 (301) 975-4037

For specific standards-related information from NCSCI, call (301) 975-4038, -4040 , or -4036 .

For up-to-date information on foreign proposed regulations, call the GATT Hotline (301) 975-4041. 


\section{STANDARDS CODE SIGNATORIES}

$\begin{array}{ll}\text { Argentina (a) } & \text { Japan } \\ \text { Austria } & \text { Korea } \\ \text { Belgium } & \text { Luxembourg (b) } \\ \text { Brazil } & \text { Mexico (b) } \\ \text { Canada } & \text { Netherlands } \\ \text { Chile (b) } & \text { New Zealand } \\ \text { Czechoslovakia } & \text { Norway } \\ \text { Denmark } & \text { Pakistan (b) } \\ \text { Egypt (b) } & \text { Philippines } \\ \text { European Economic } & \text { Portugal (b) } \\ \text { Community (EEC) } & \text { Romania (a) (c) } \\ \text { Finland } & \text { Rwanda (angapore } \\ \text { France } & \text { Sinain } \\ \text { Federal Republic of } & \text { Sparmany } \\ \text { Greece (b) } & \text { Sweden } \\ \text { Hong Kong } & \text { Tunisia } \\ \text { Hungary } & \text { United Kingdom } \\ \text { India } & \text { United States } \\ \text { Ireland } & \text { Yugoslavia } \\ \text { Israel (b) } & \\ \text { Italy } & \end{array}$

(a) - the country has not yet ratified the standards code and has not reported any proposed regulations to the GATT secretariat. (b) - the country has not reported any proposed regulations to the GATT secretariat.

(c) - the country has not established an inquiry point for standards information and has not reported any proposed regulations to the GATT secretariat. 


\section{TABLE I}

Notifying Countries and

Number of GATT Notifications Issued in 1990

Signatory

Austria
Belgium
Brazil
Canada
Czechoslovakia
Denmark
EEC
Finland
France
Germany
Hong Kong
Hungary
India
Japan
Korea
Netherlands
New Zealand
Norway
Philippines
Singapore
Sweden
Switzerland
United Kingdom
United States
Yugoslavia

Number of Notifications

$\begin{array}{r}22 \\ 11 \\ 2 \\ 8 \\ 47 \\ 13 \\ 17 \\ 26 \\ 3 \\ 7 \\ 2 \\ 2 \\ 1 \\ 32 \\ 2 \\ 12 \\ 32 \\ 9 \\ 17 \\ 1 \\ 34 \\ 6 \\ 1 \\ 30 \\ 25 \\ \hline\end{array}$

22 1 8 17 26 7 2 2 2 2 7 1 1 25

Total 362 
TABLE II

List of U.S. Notifications

$\begin{array}{ll}\text { TBT/NOTIF. } & \text { AGENCY } \\ 90.40 & \text { FCC }(291) * \\ 90.41 & \text { NHTSA }(290) \\ 90.57 & \text { NHTSA }(292) \\ 90.60 & \text { NHTSA }(294) \\ 90.61 & \text { FDA }(293) \\ 90.62 & \text { FDA }(297) \\ 90.63 & \text { FDA }(296) \\ 90.64 & \text { FDA }(295) \\ 90.82 & \text { DOE }(298) \\ 90.129 & \text { NHTSA }(299) \\ 90.161 & \text { HUO }(301) \\ 90.162 & \text { USDA }(300) \\ 90.176 & \text { USDA }(302) \\ 90.205 & \text { FDA }(305) \\ 90.214 & \text { FOA }(309) \\ 90.215 & \text { FDA }(306) \\ 90.216 & \text { FDA }(307) \\ 90.217 & \text { FDA }(306) \\ 90.223 & \text { CPSC }(304) \\ 90.224 & \text { CPSC }(303) \\ 90.228 & \text { NHTSA }(310) \\ 90.248 & \text { NHTSA }(311) \\ 90.249 & \text { DOT }(312) \\ 90.262 & \text { DOT }(313) \\ 90.295 & \text { EPA }(314) \\ 90.311 & \text { CPSC }(315) \\ 90.312 & \text { NHTSA }(316) \\ 90.320 & \text { CPSC }(318) \\ 90.321 & \text { CPSC }(317) \\ 90.353 & \text { FOA }(319)\end{array}$

PROOUCT

COROLESS TELEPHONES

PASSENGER CARS

SCHOOL BUSES

TRAILER PNEUMATIC BRAKE SYSTEMS

FDDD PACKAGING MATERIALS

ANIMAL FEED

FOOO LABELING

DIAGNOSTIC X-RAY SYSTEMS

FLUDRESCENT LAMP BALLASTS

POWER DPERATED WINDOW SYSTEMS

PLYWOOO PANELS

MEAT \& POULTRY PROOUCTS

UNCURED MEAT PATTIES

BDTTLED WATER

FOOO LABELING

FDOD LABELING

FDDD LABELING

FOOO LABELING

BALLDONS. SMALL BALLS ANO MARBLES

TOYS ANO ARTICLES FOR CHILDREN

SAFETY BELTS

MOTOR VEHICLE TIRES \& RIMS

MARINE EQUIPMENT \& COMPONENTS

TANK CAR TANKS

MAJOR HOUSEHOLD APPLIANCES

HDUSEHDLD SUBSTANCES

MOTOR VEHICLES

CRIB TOYS

INFANT CUSHIONS \& FILLOWS

MEOICAL OEVICES 


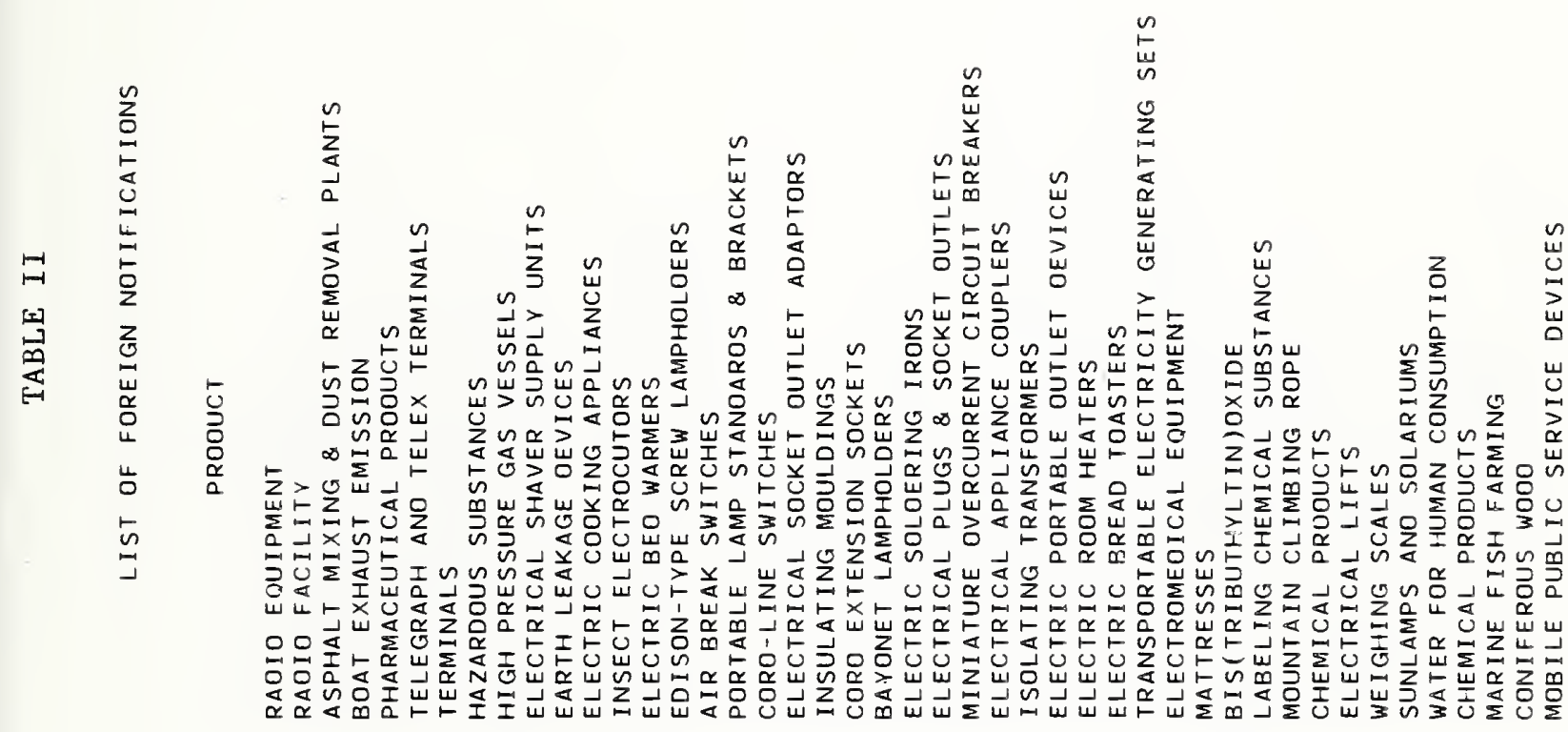

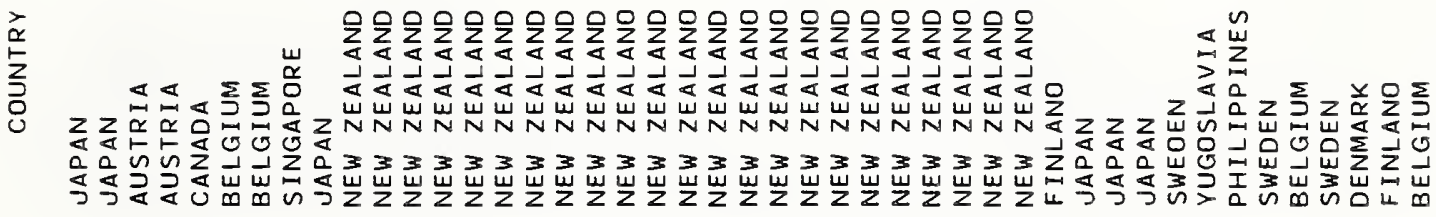

点 -

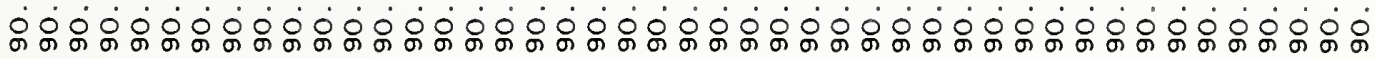



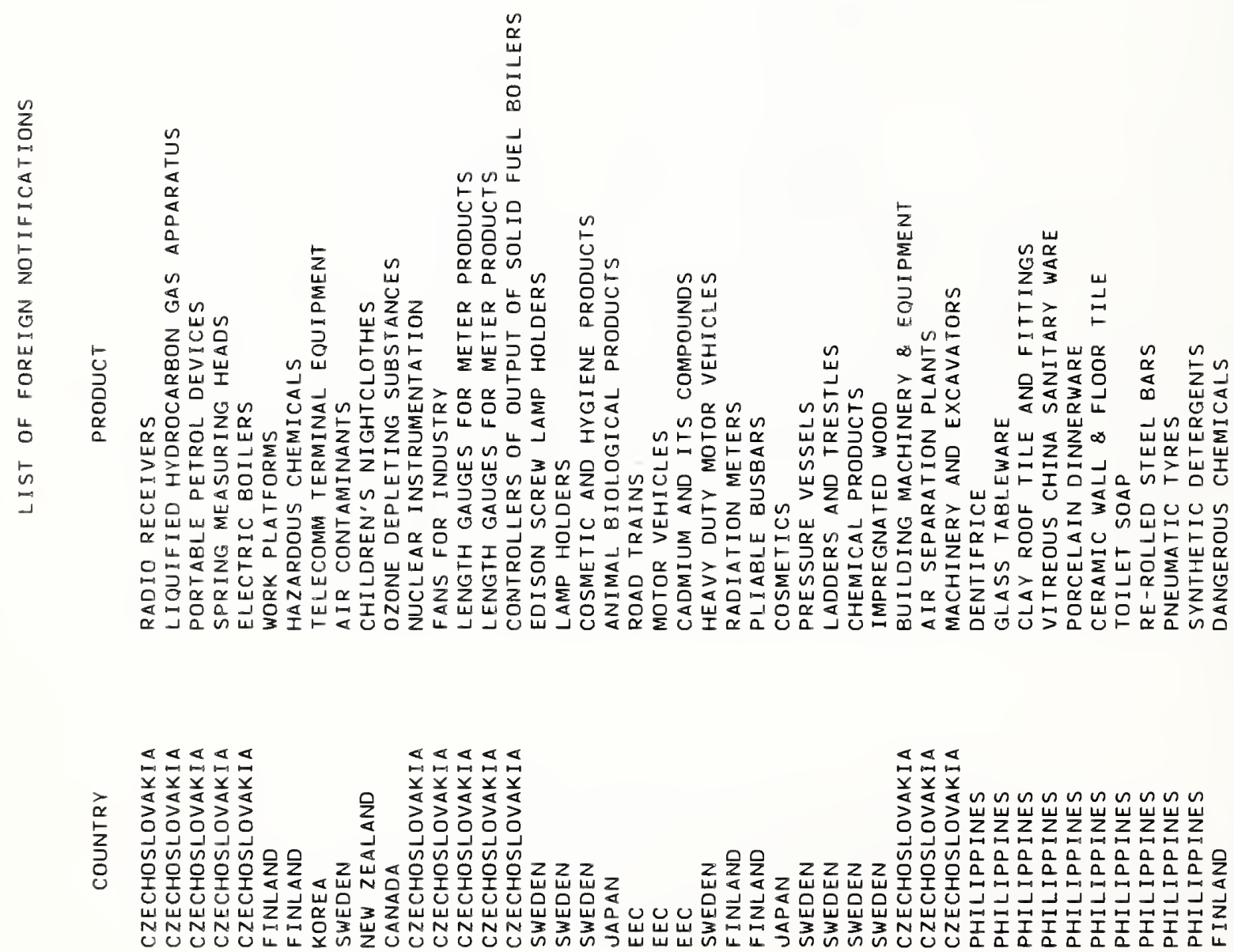

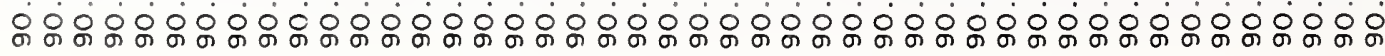



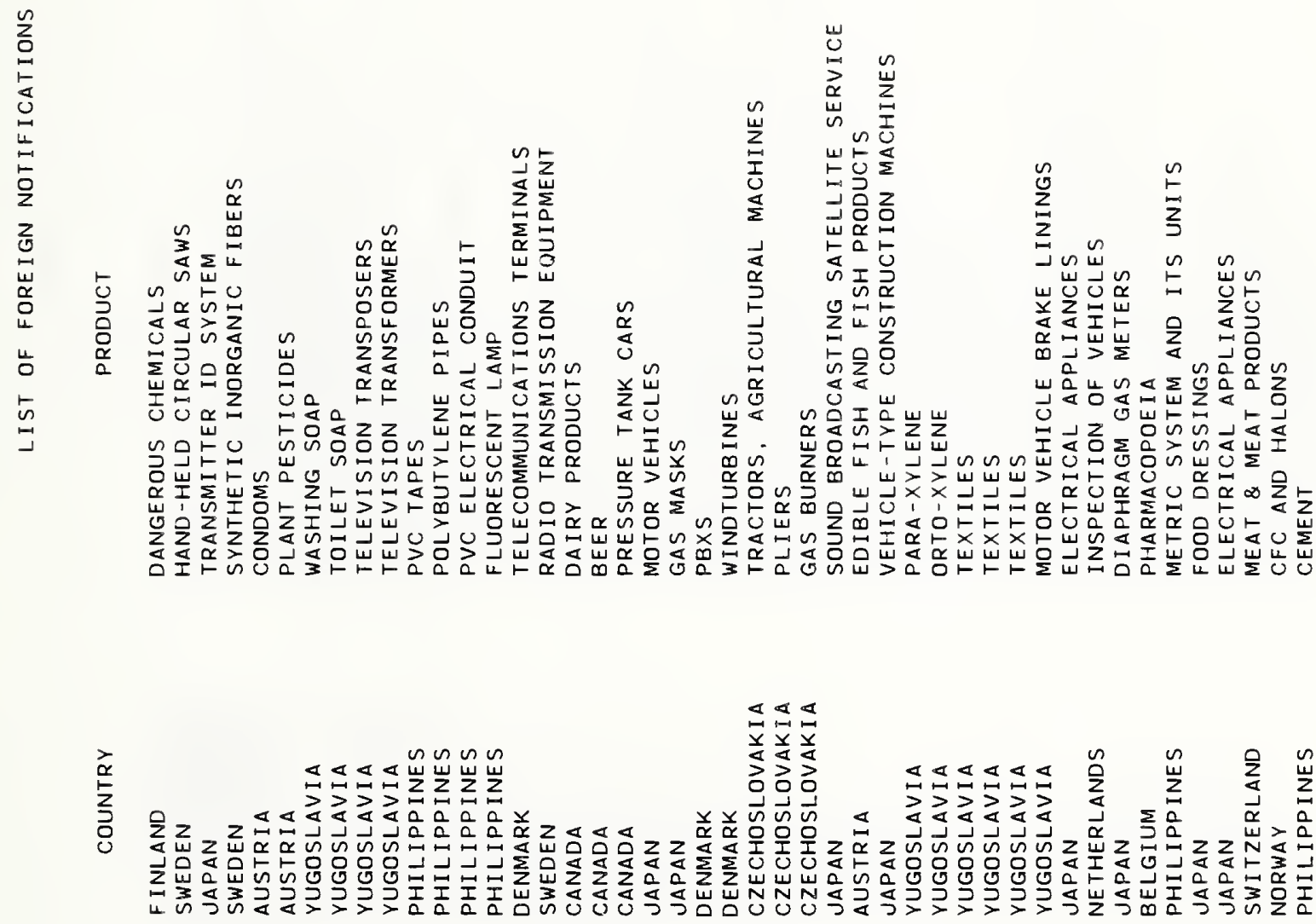

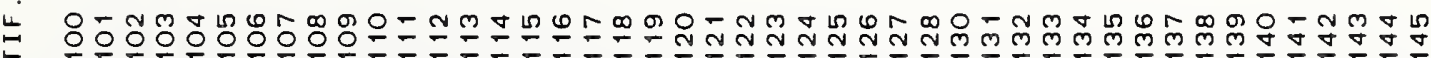

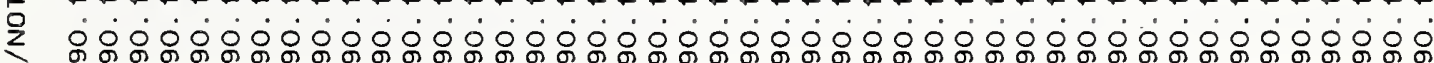
$\stackrel{\circ}{\circ}$
} 

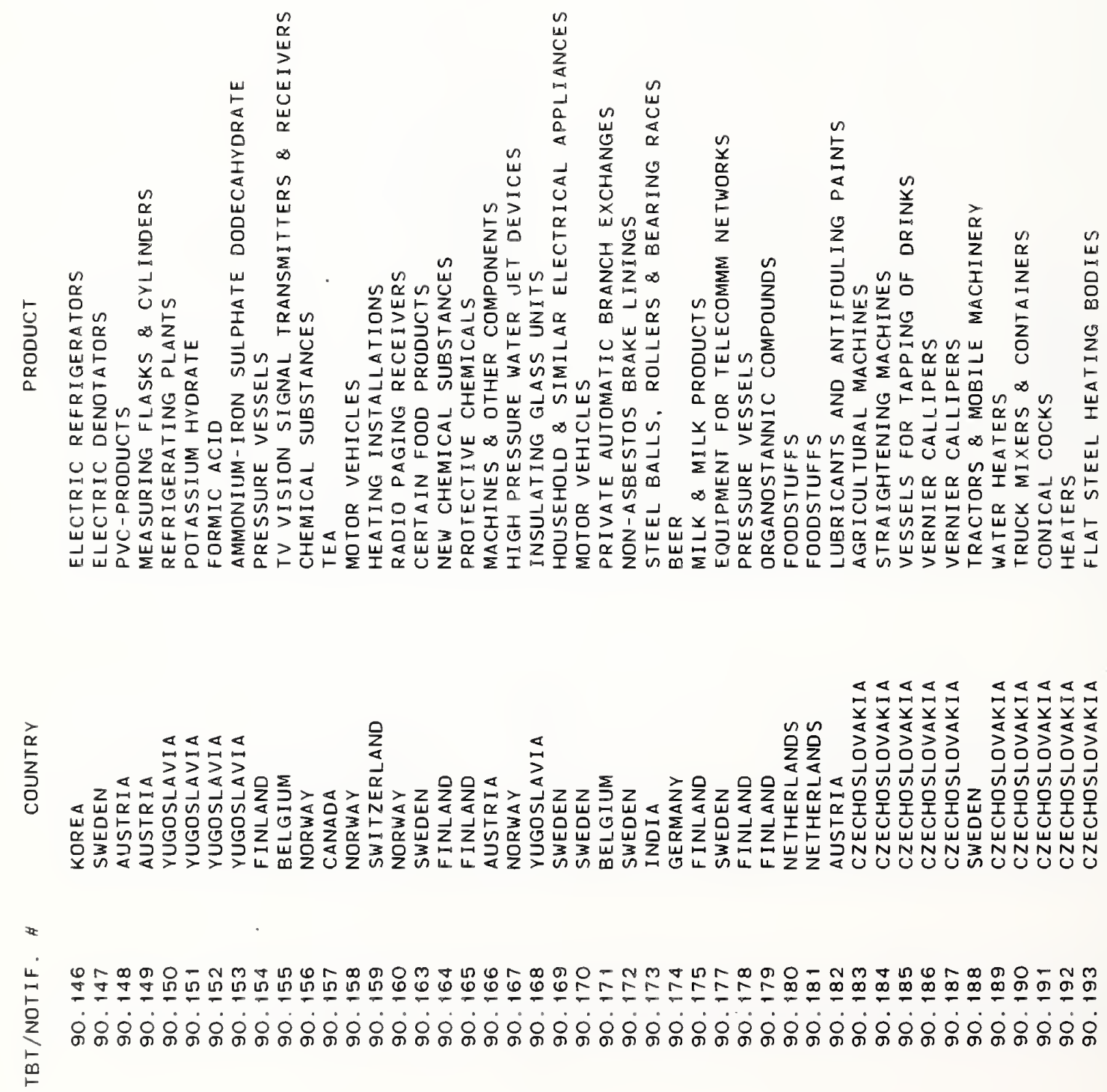

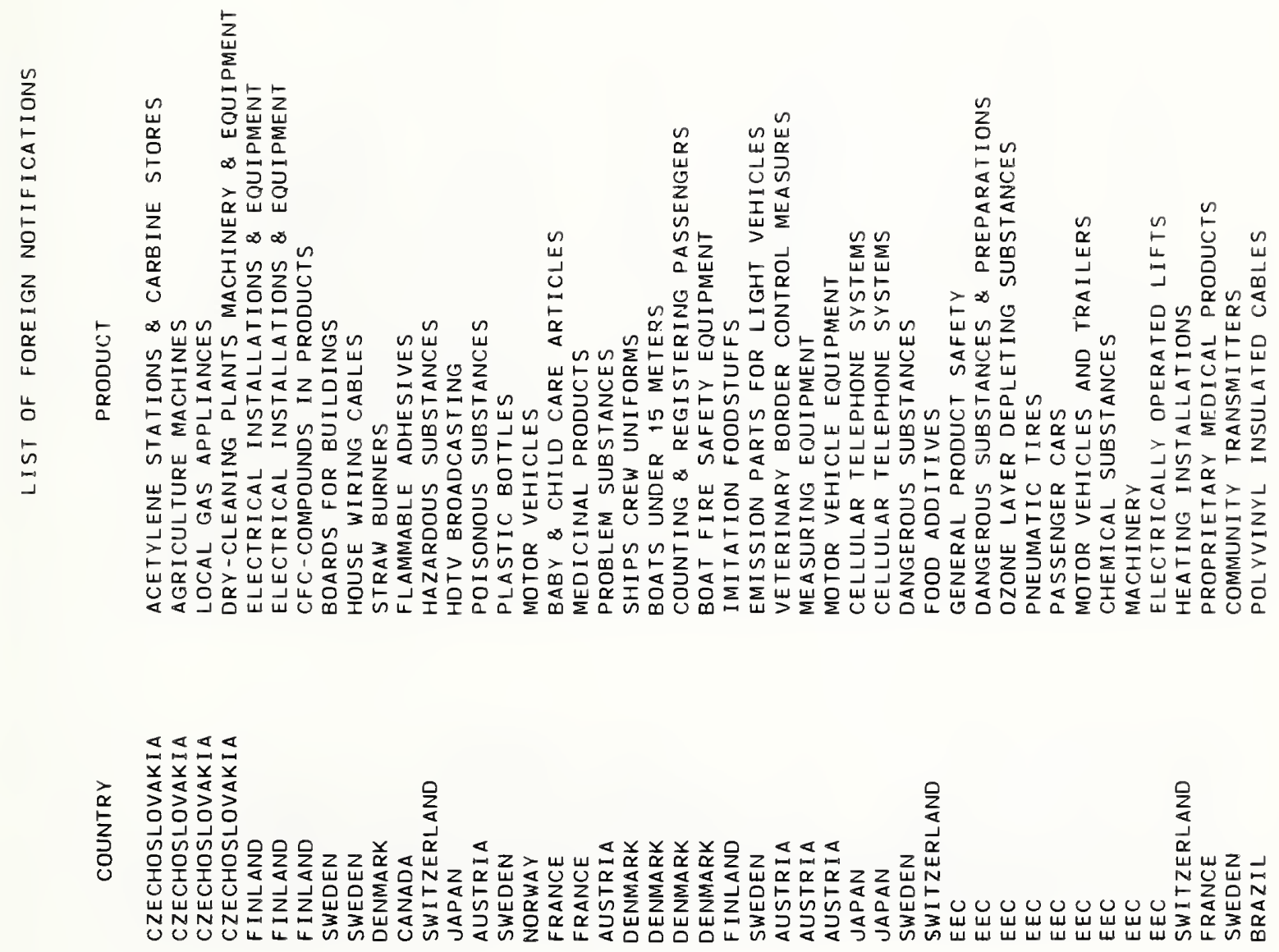

草

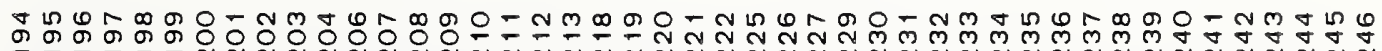

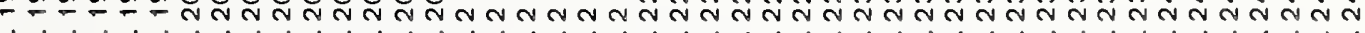

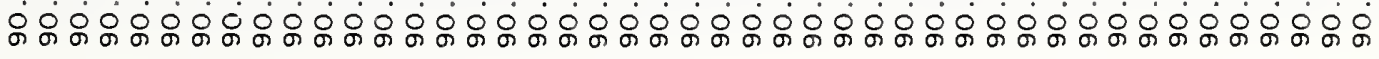



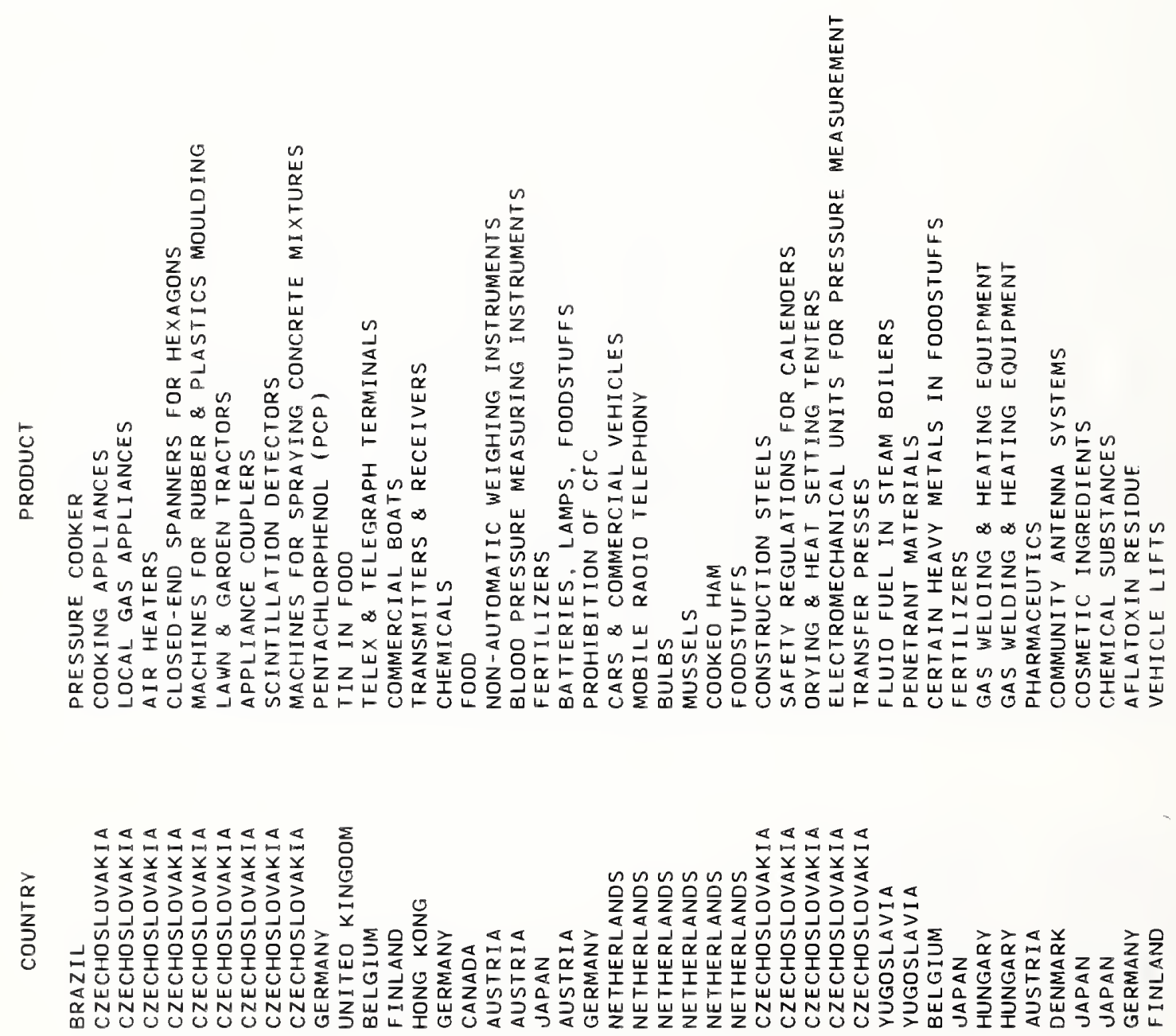

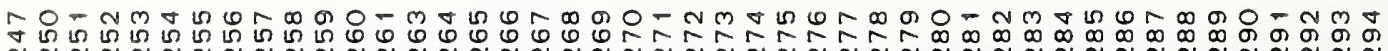

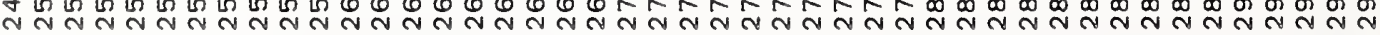

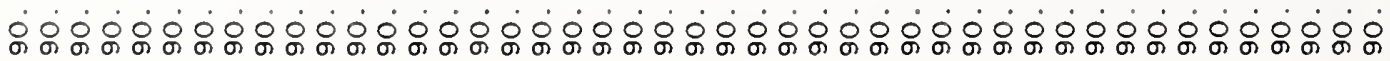



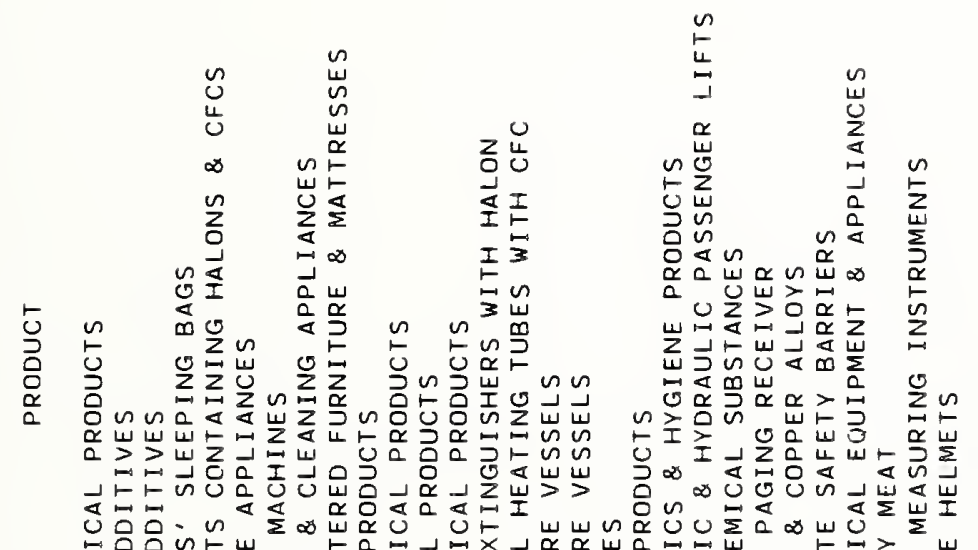

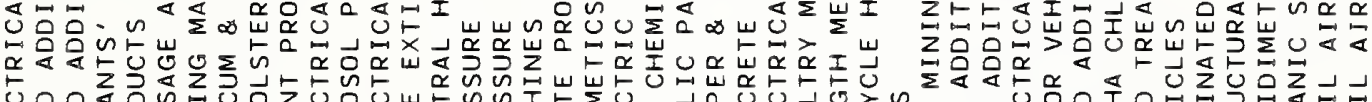

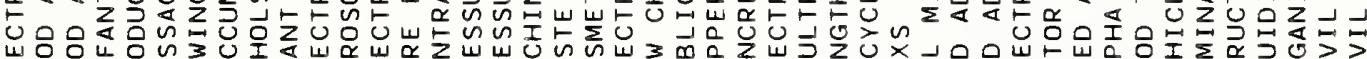

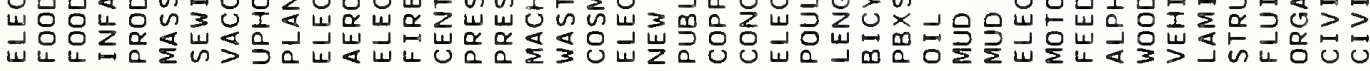

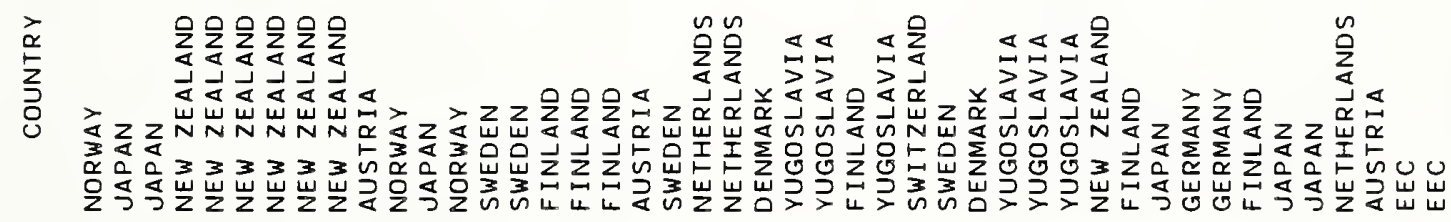

மก⿻冂土-

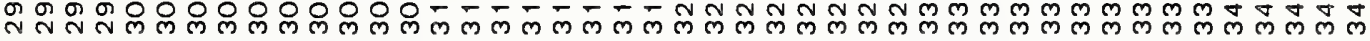

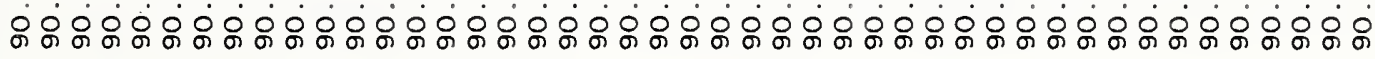



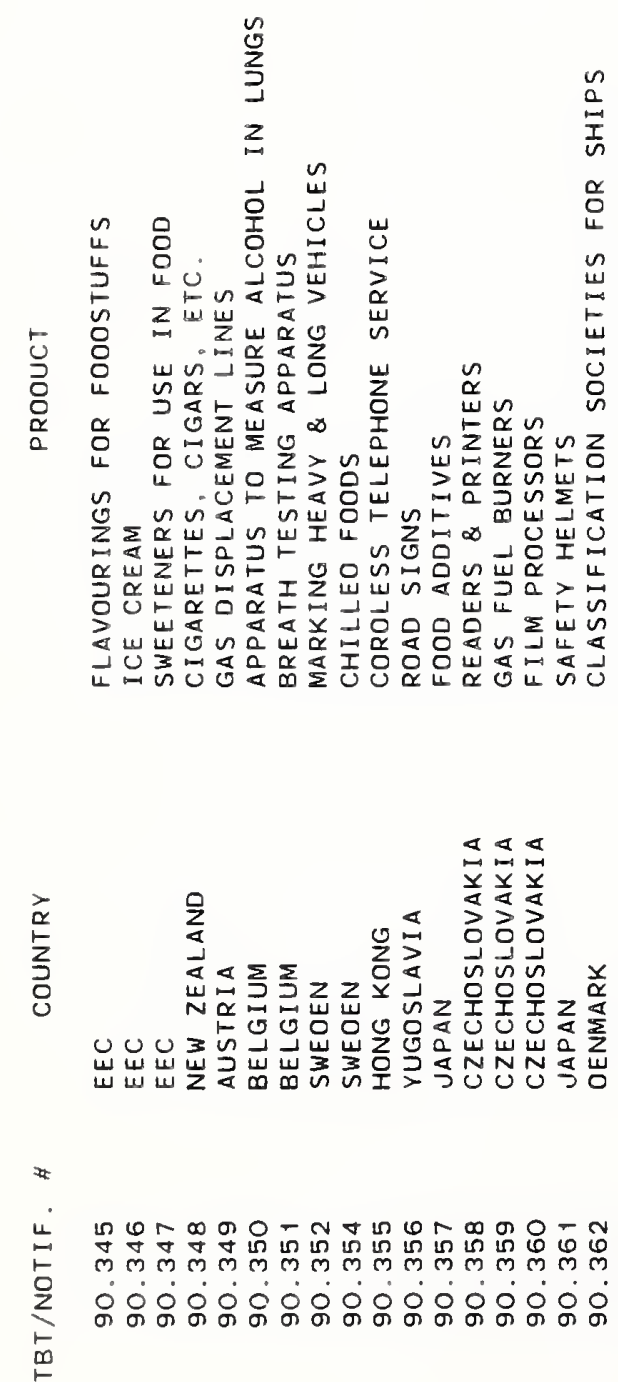


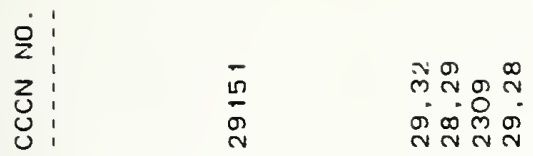

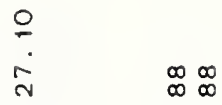

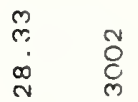

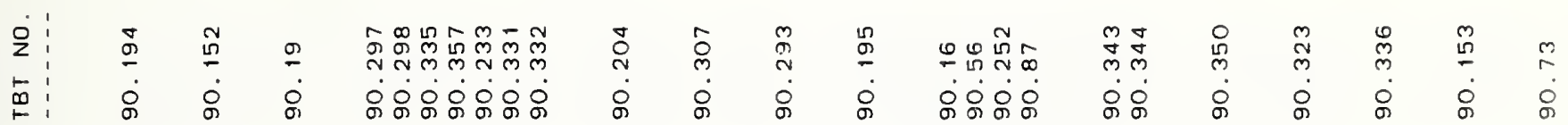

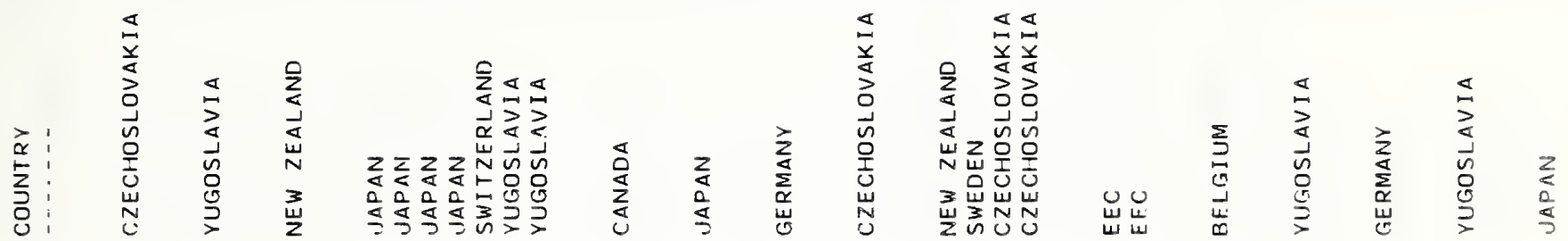

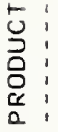

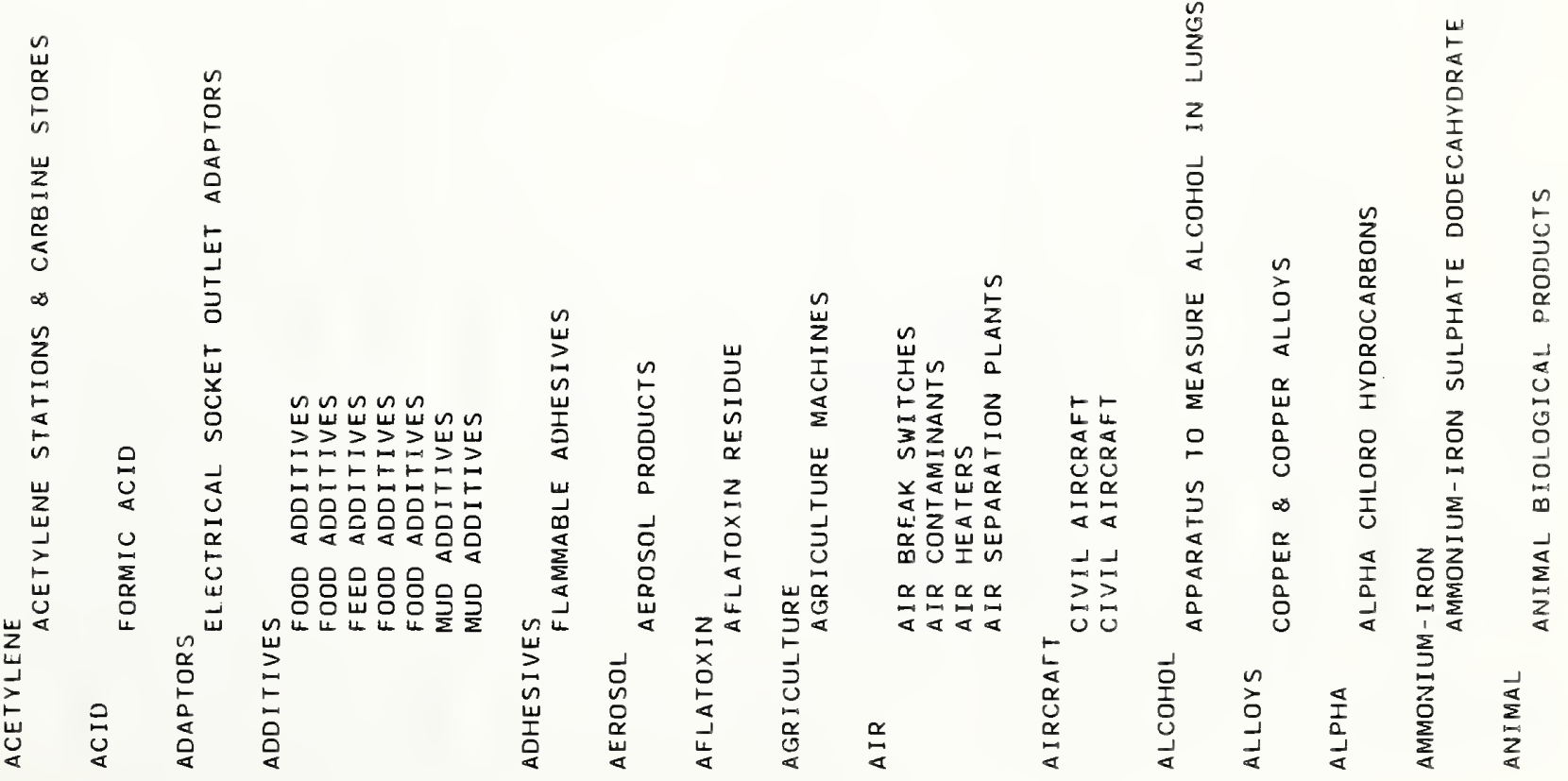




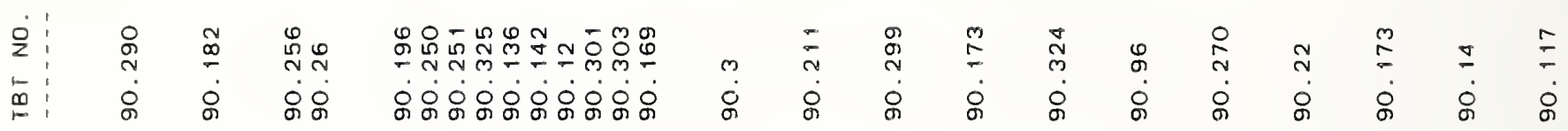

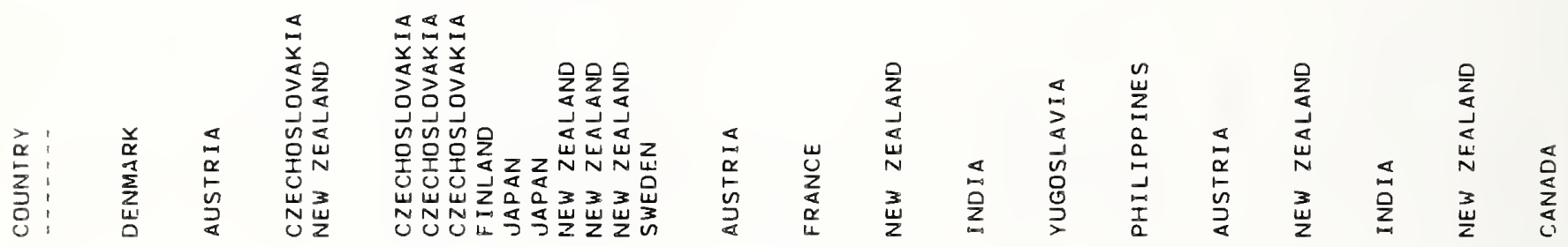

:

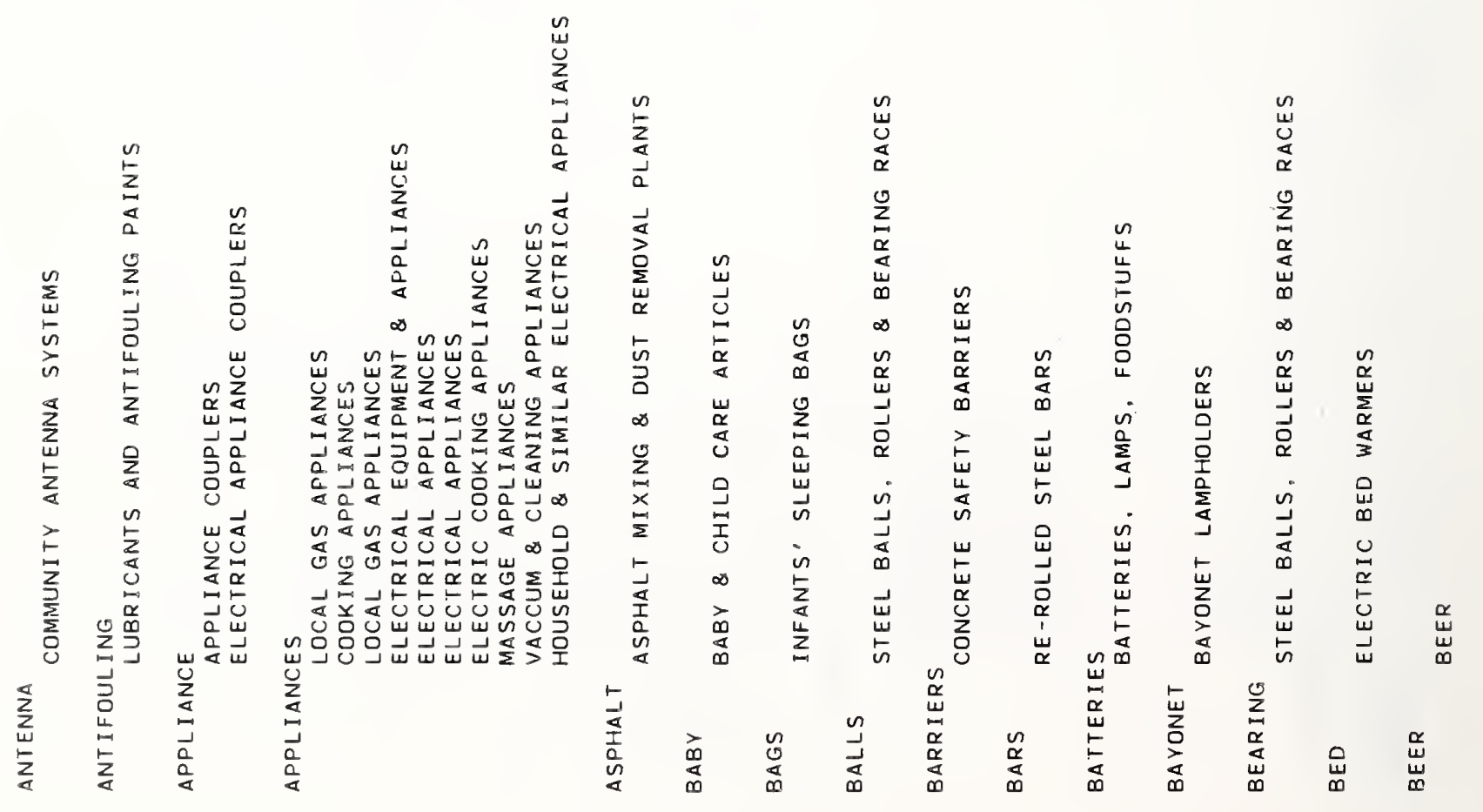




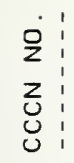

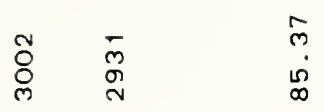

용요

$\stackrel{m}{*}$

串

品

कि

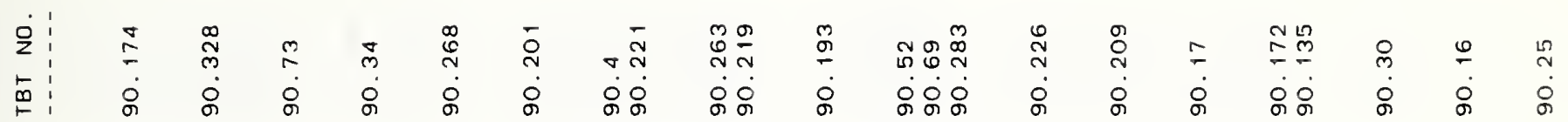

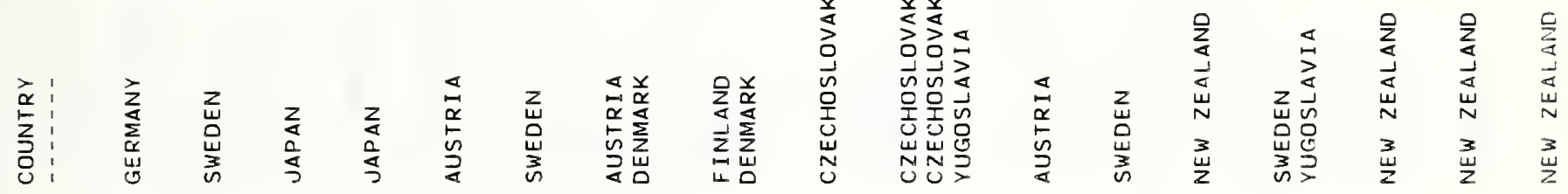

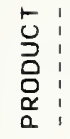

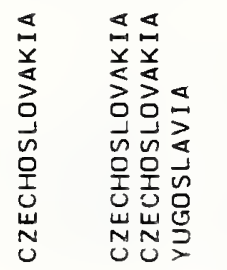

足

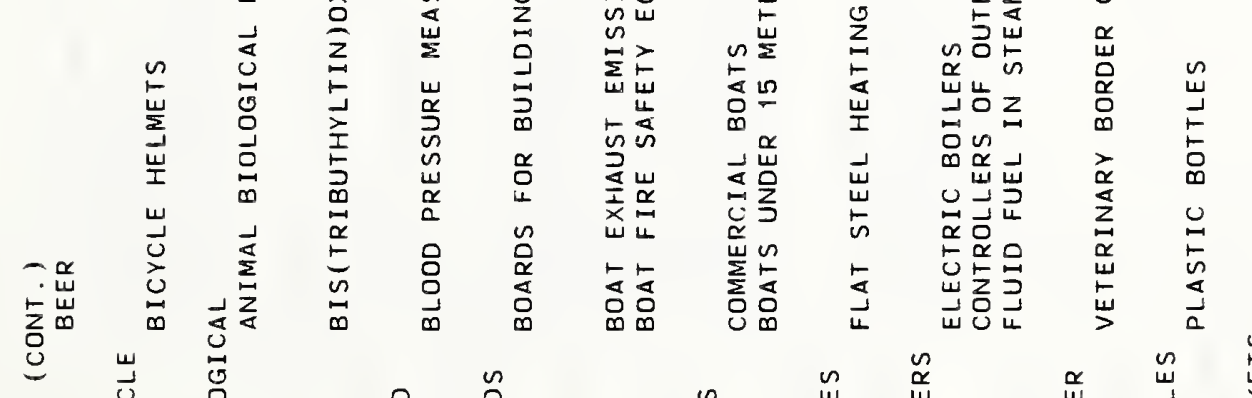

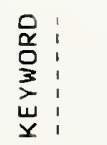

噌

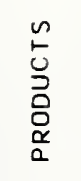

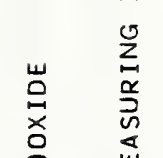

5 n

究总

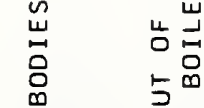

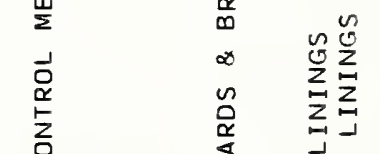

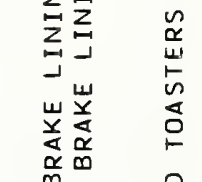

œ嵌

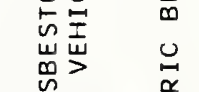

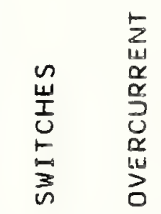

妾

尔品

峁

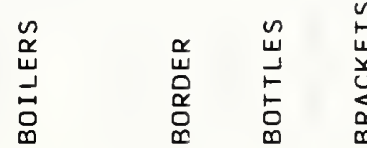

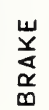

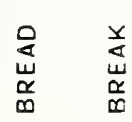

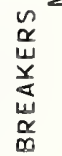


管:

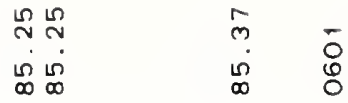

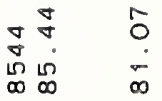

$\begin{array}{ll}m & \ddot{0} \\ \dot{0} & \dot{n}\end{array}$

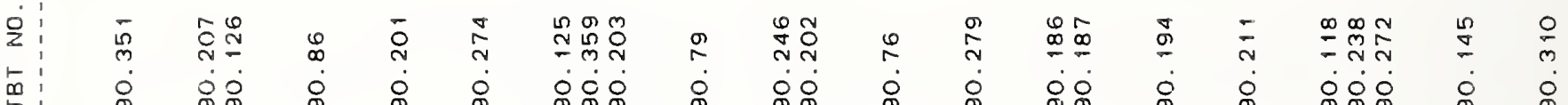

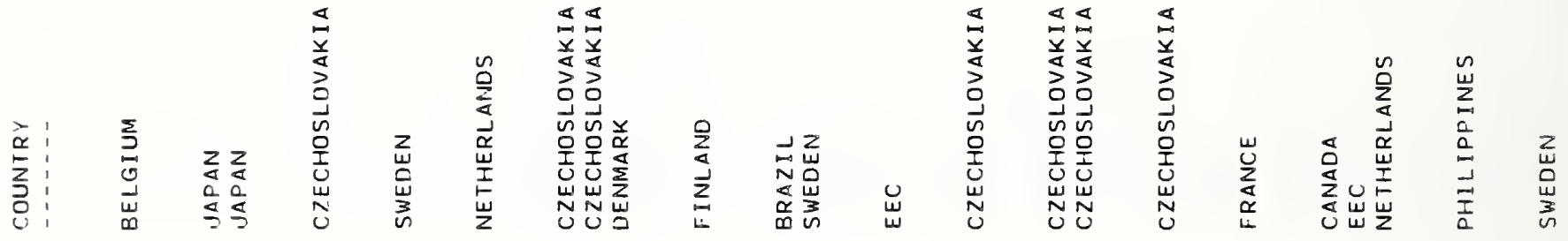

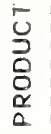

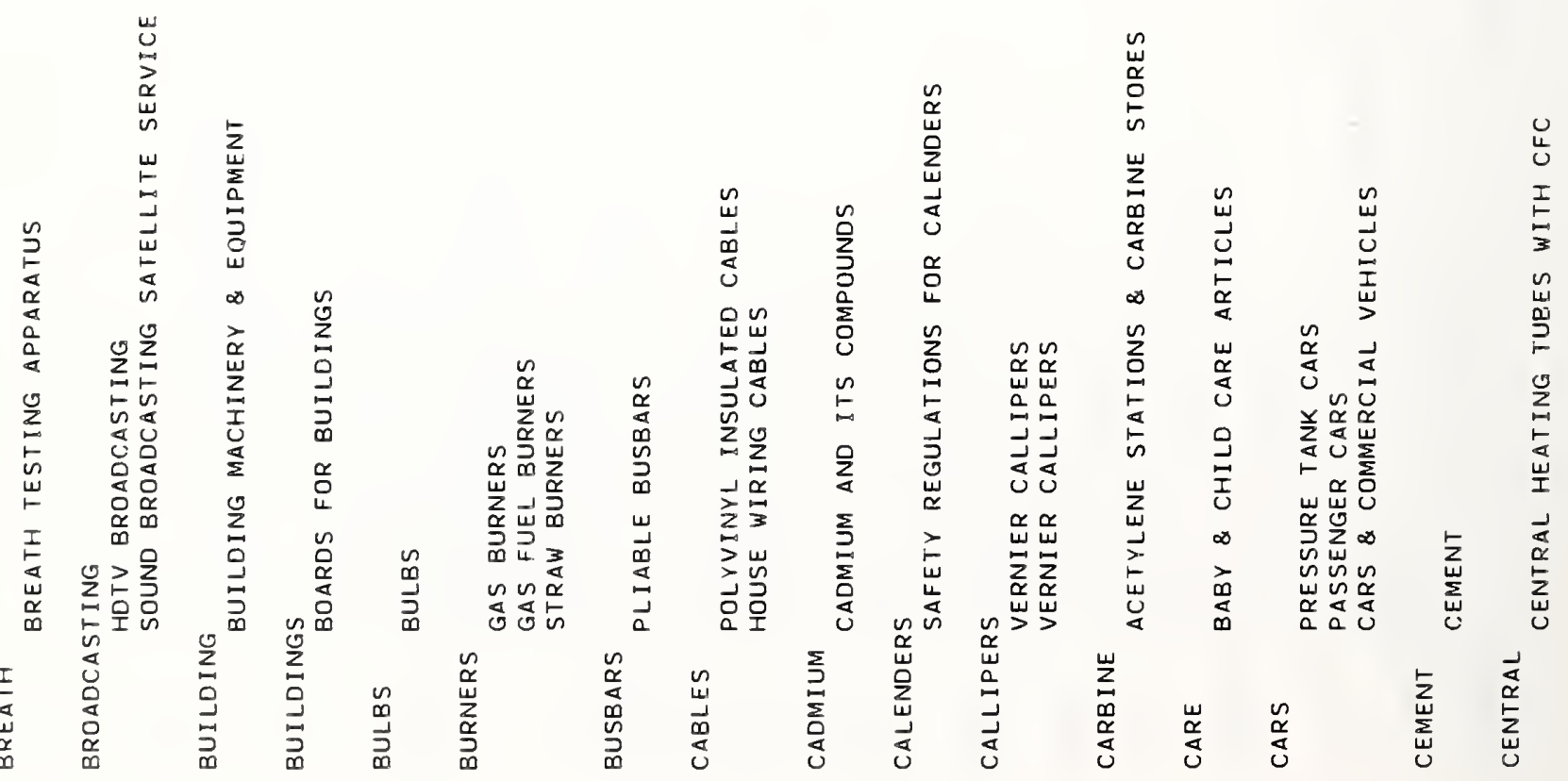




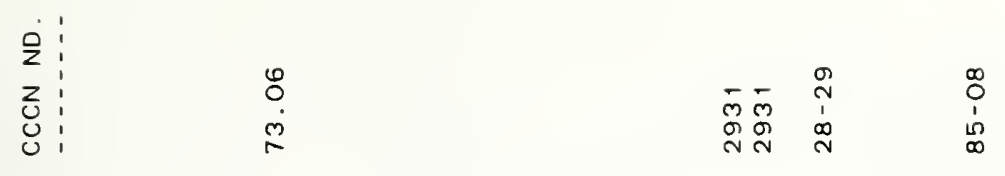

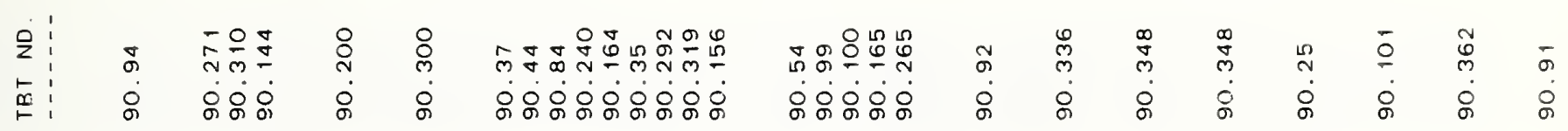

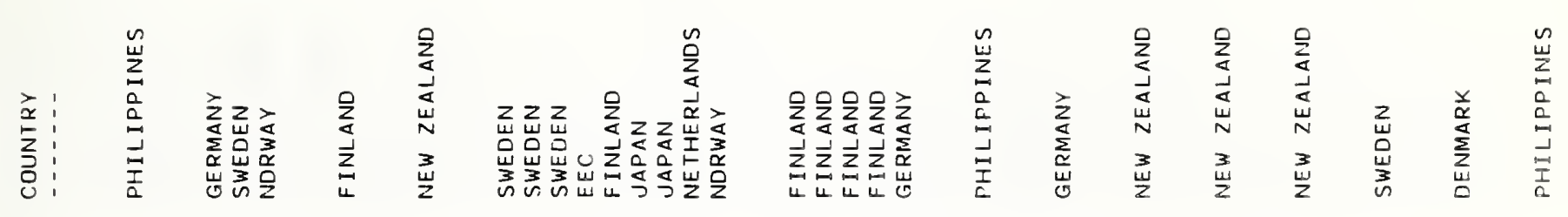

:

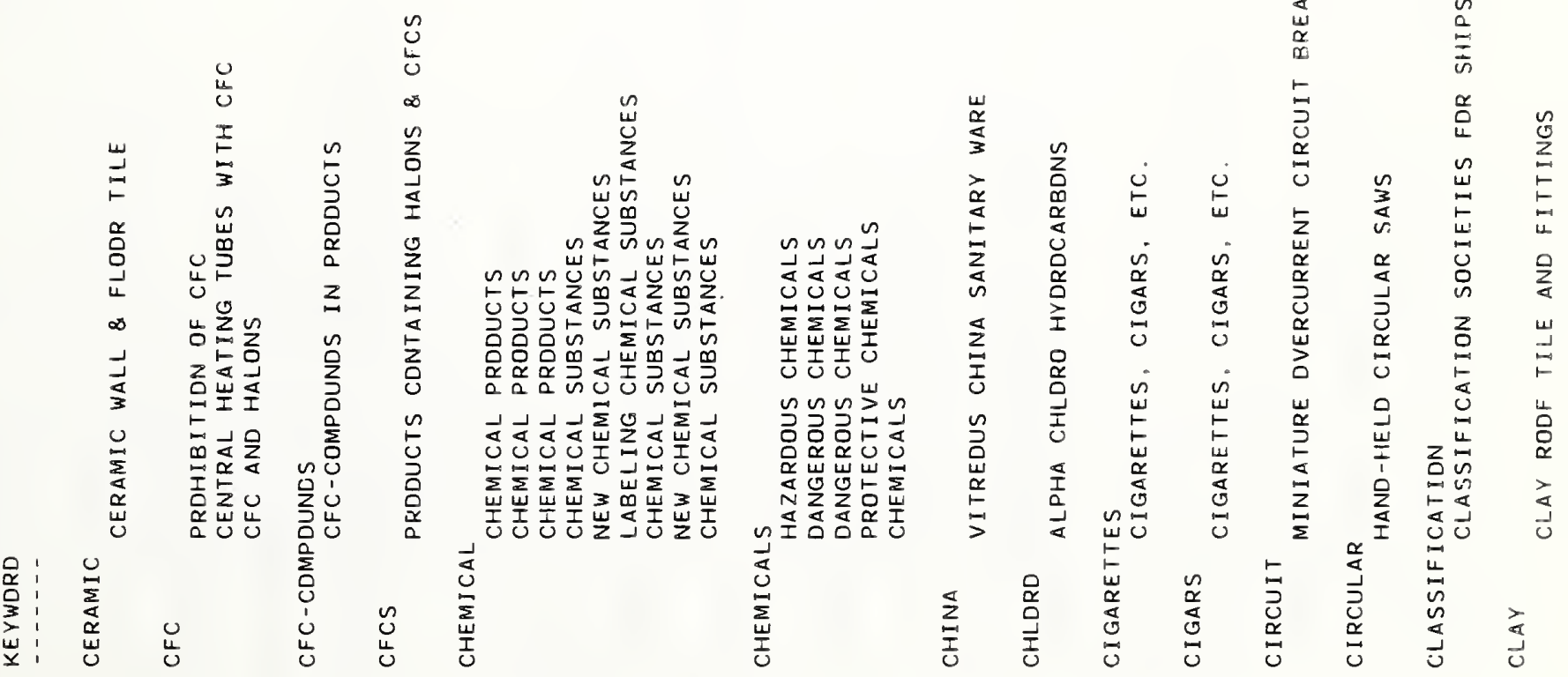


旁:

0
0
0
0

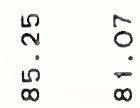

$\underset{\infty}{\stackrel{0}{\infty}}$

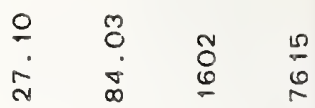

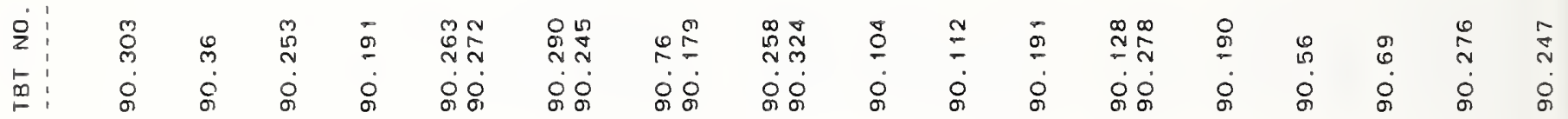

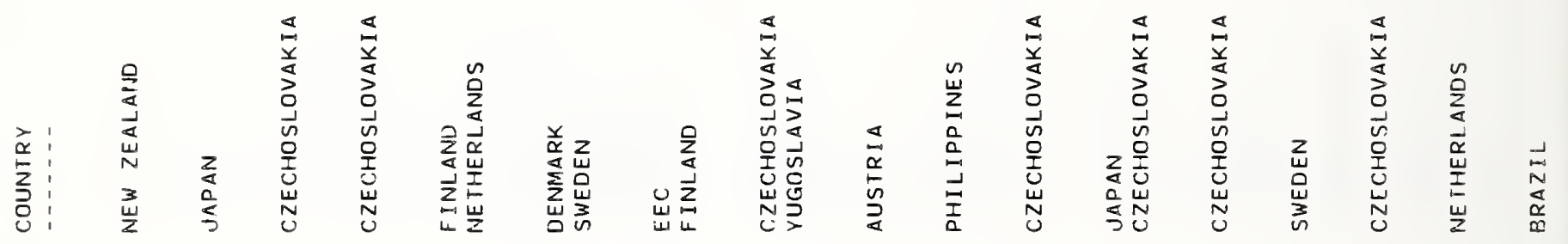

\begin{tabular}{l:c}
$\mathfrak{S}$ & $\vdots$ \\
\hdashline & $\vdots$ \\
\hdashline & $:$
\end{tabular}

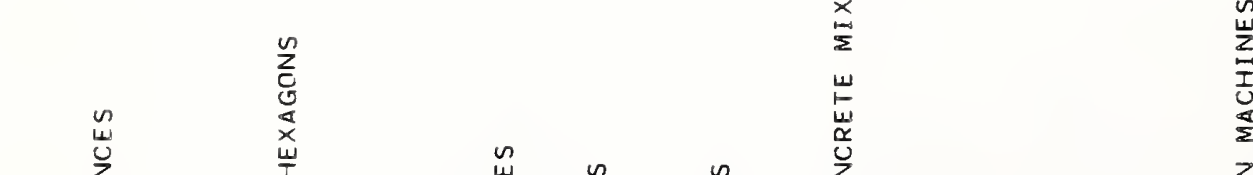

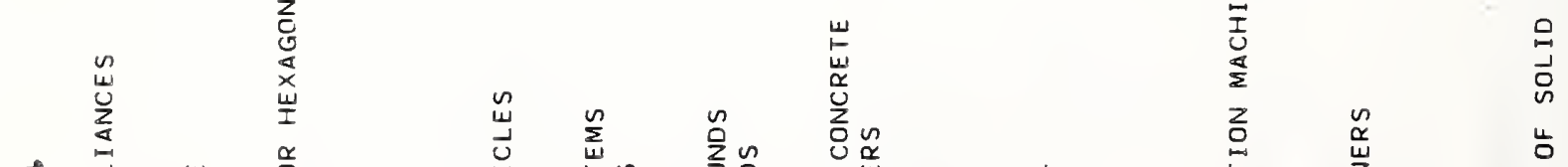

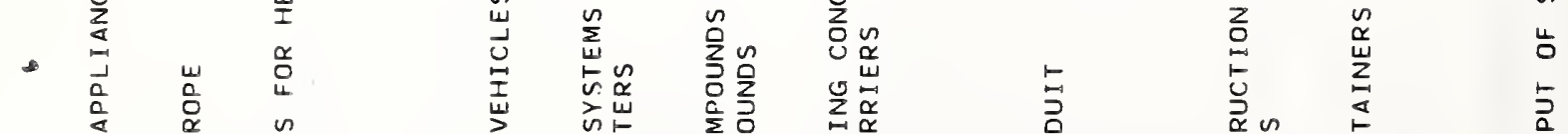

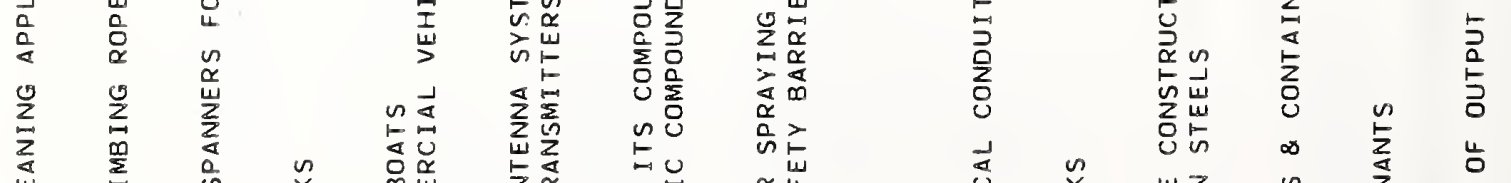

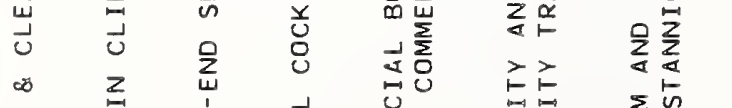

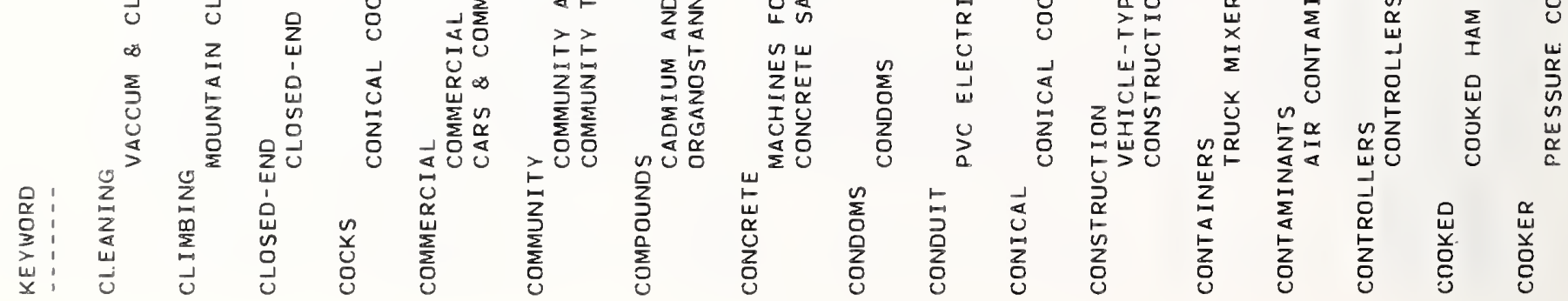

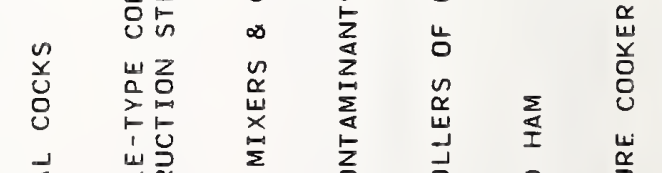

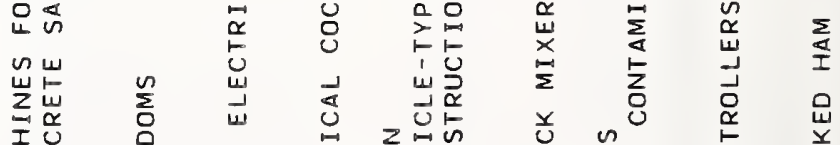




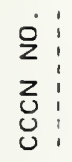

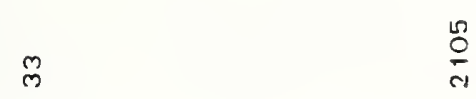

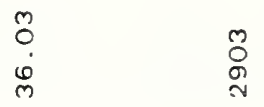

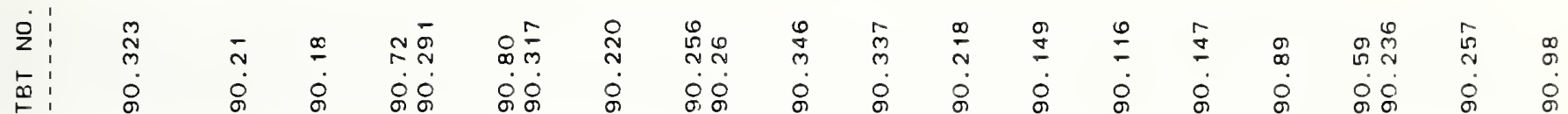

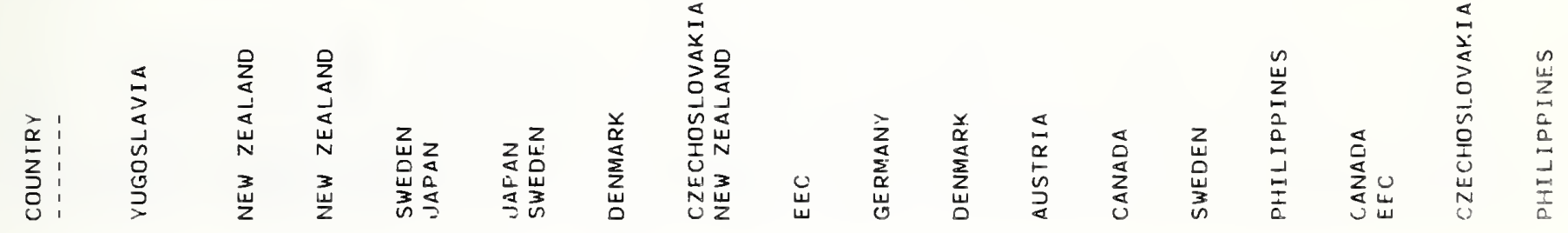

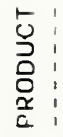

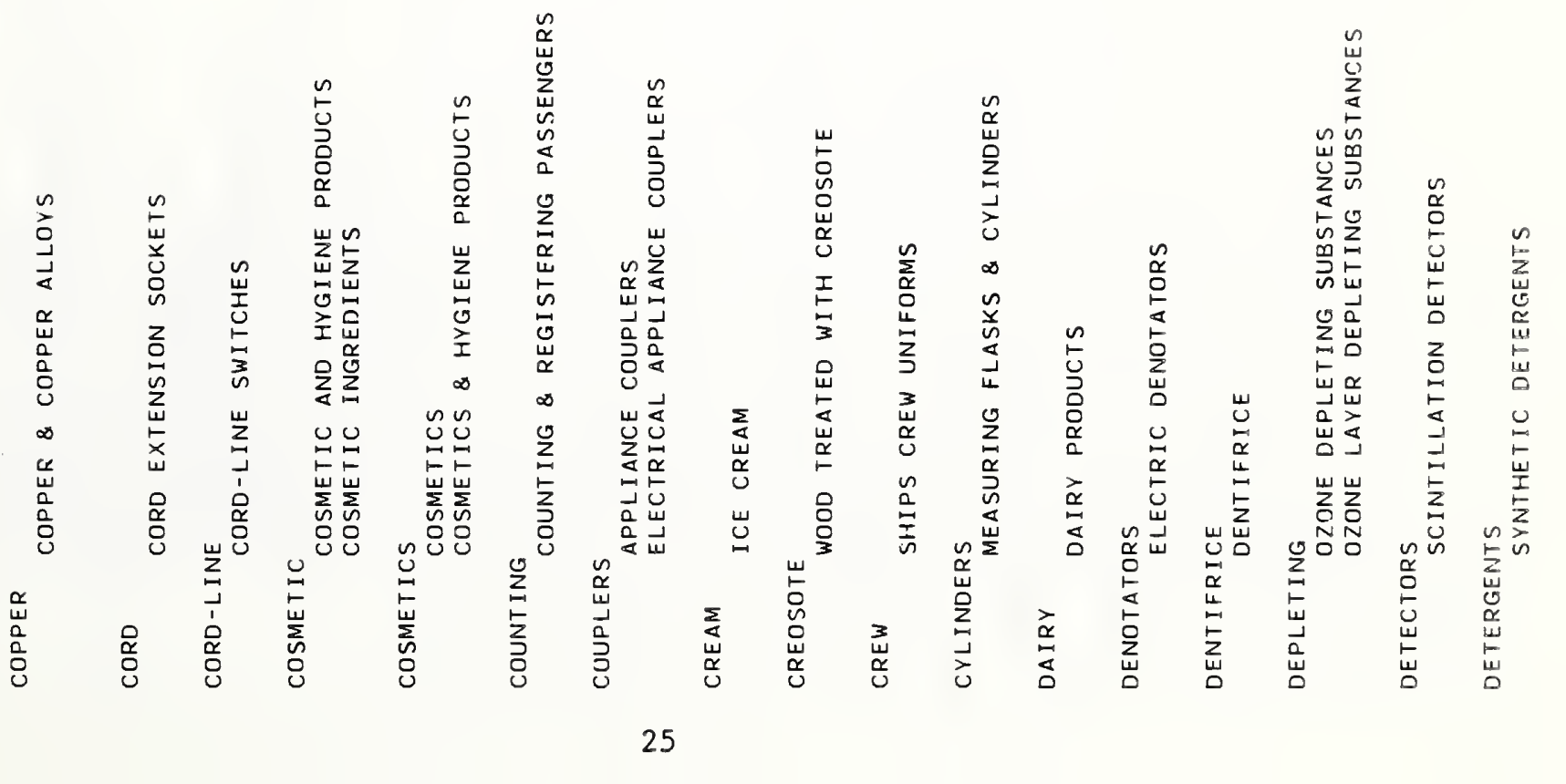




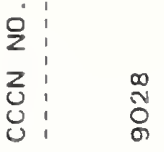

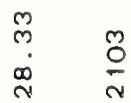

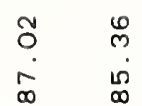

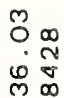

융은

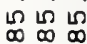

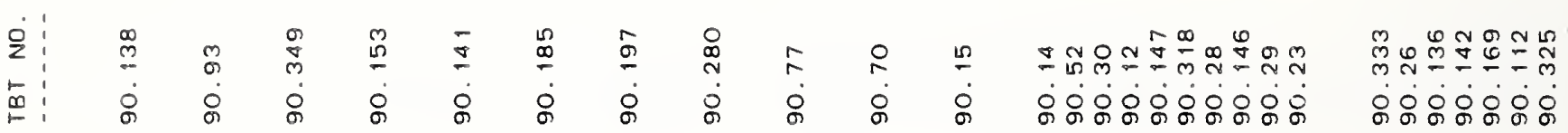

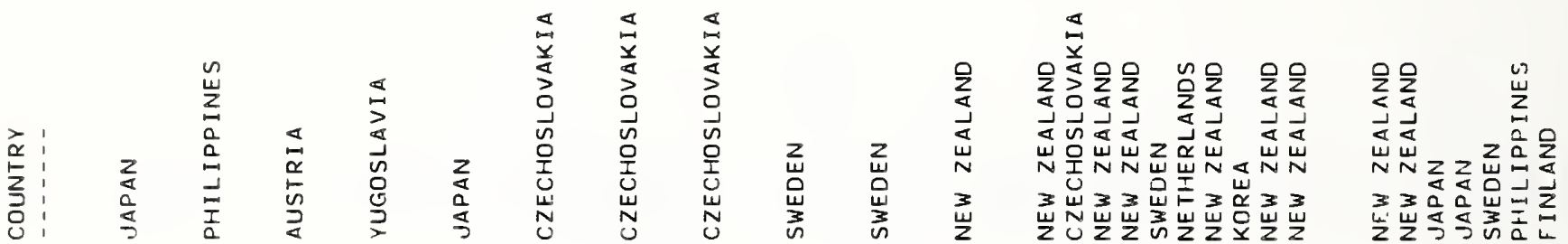

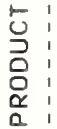

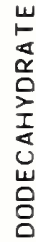

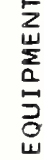

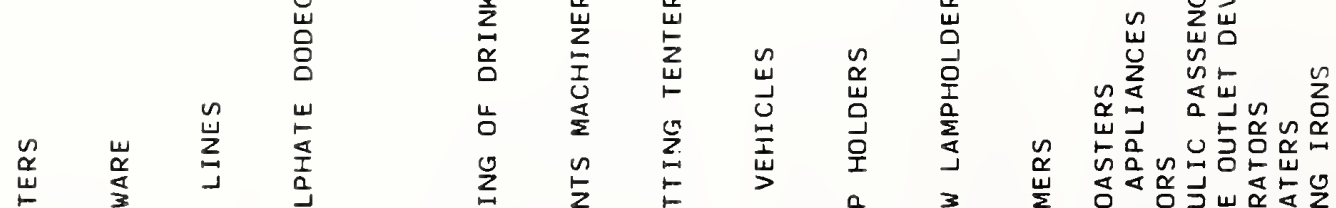

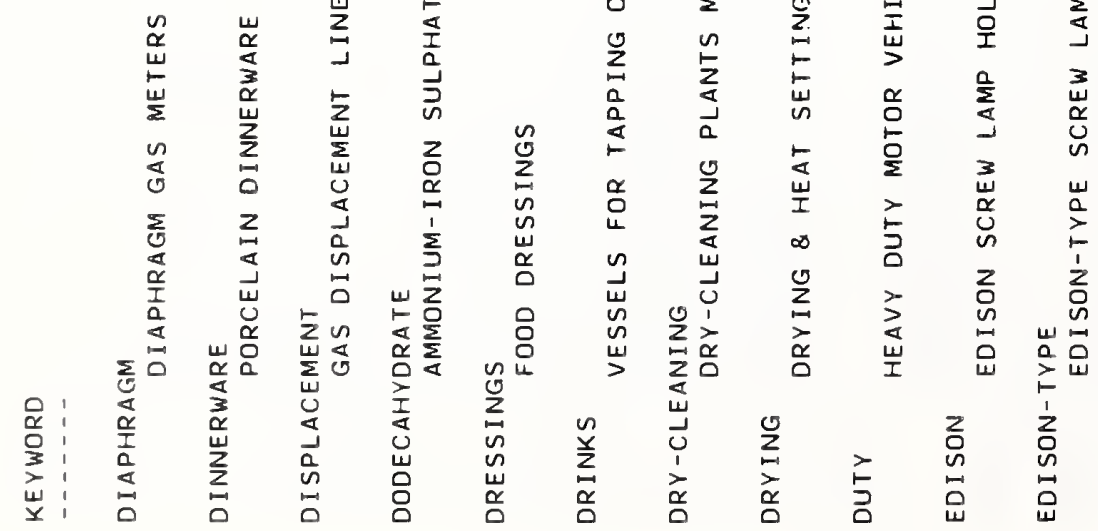

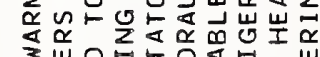

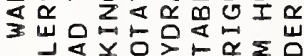

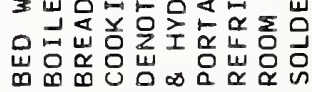

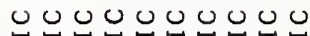

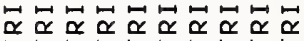

는ㄷㄴㄷㄴㄷㄴ

出出出出出出出

u

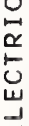

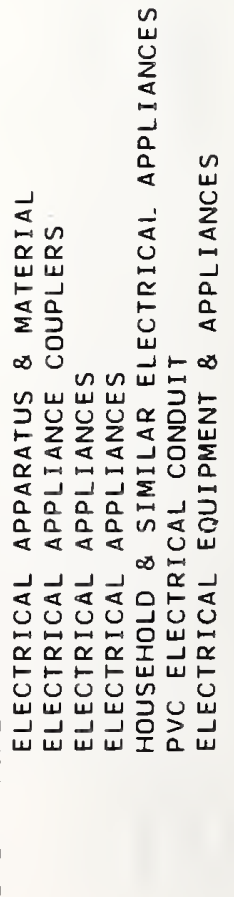


:

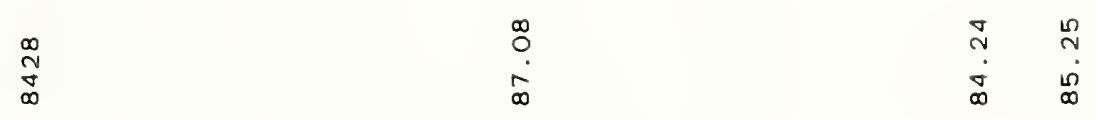

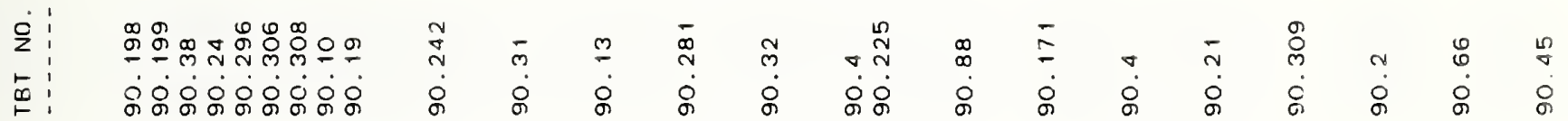

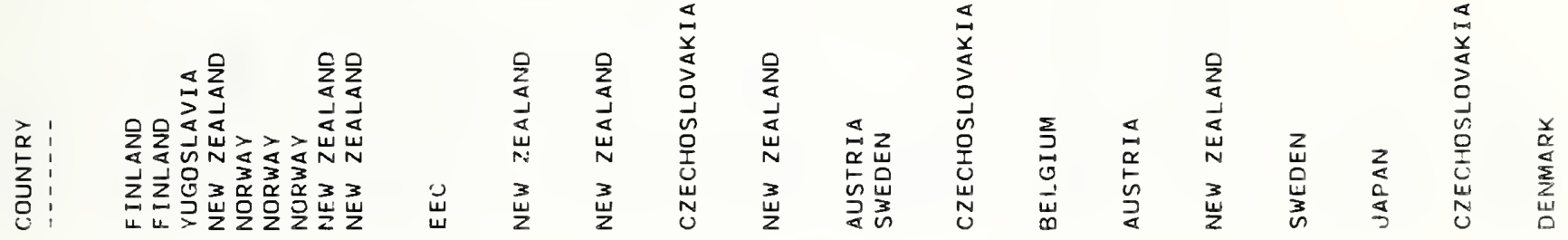

:

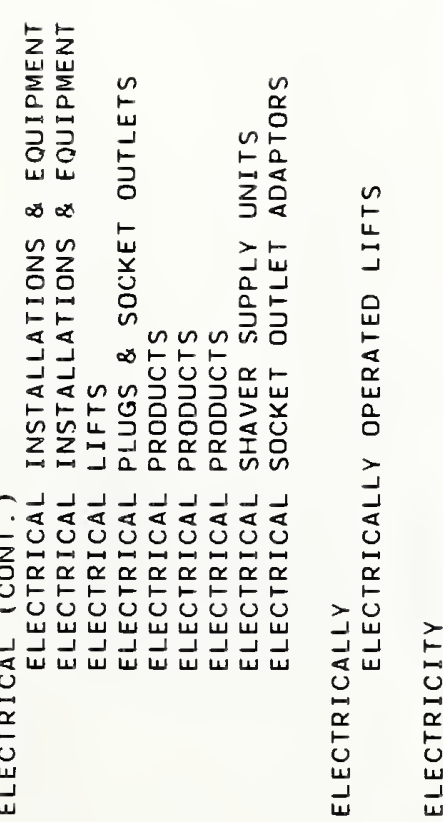

岕

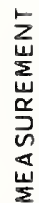

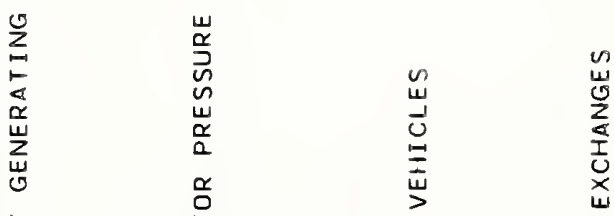

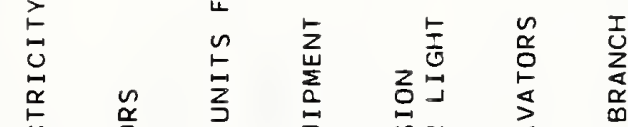

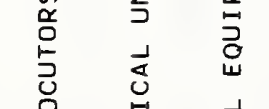

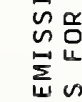

袋嵒

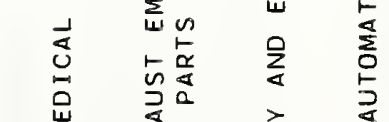

过岂

秀要

坴云

豈

皆

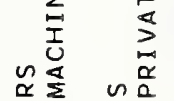

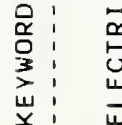

zo

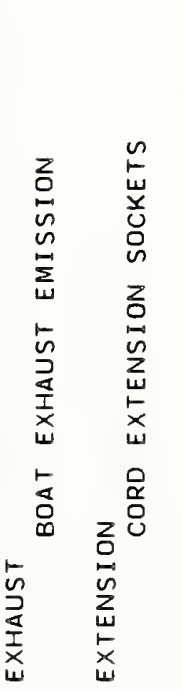

ż

5

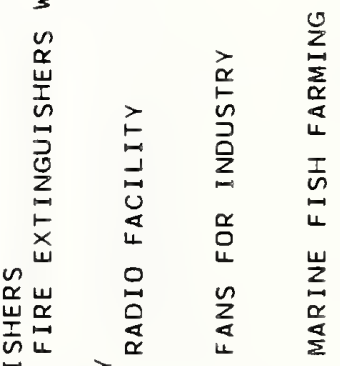

点

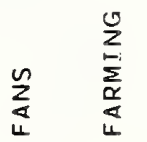




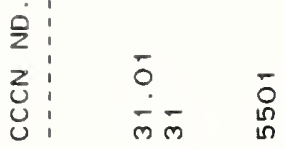

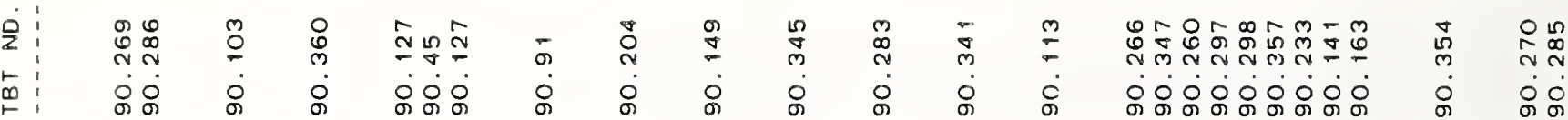

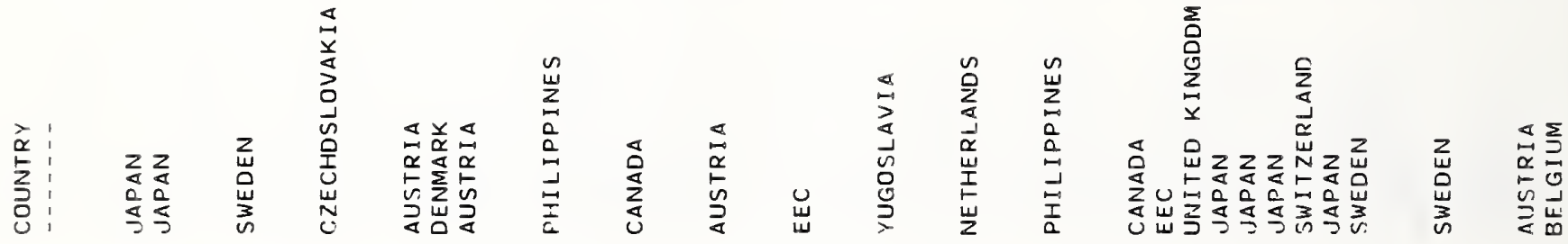

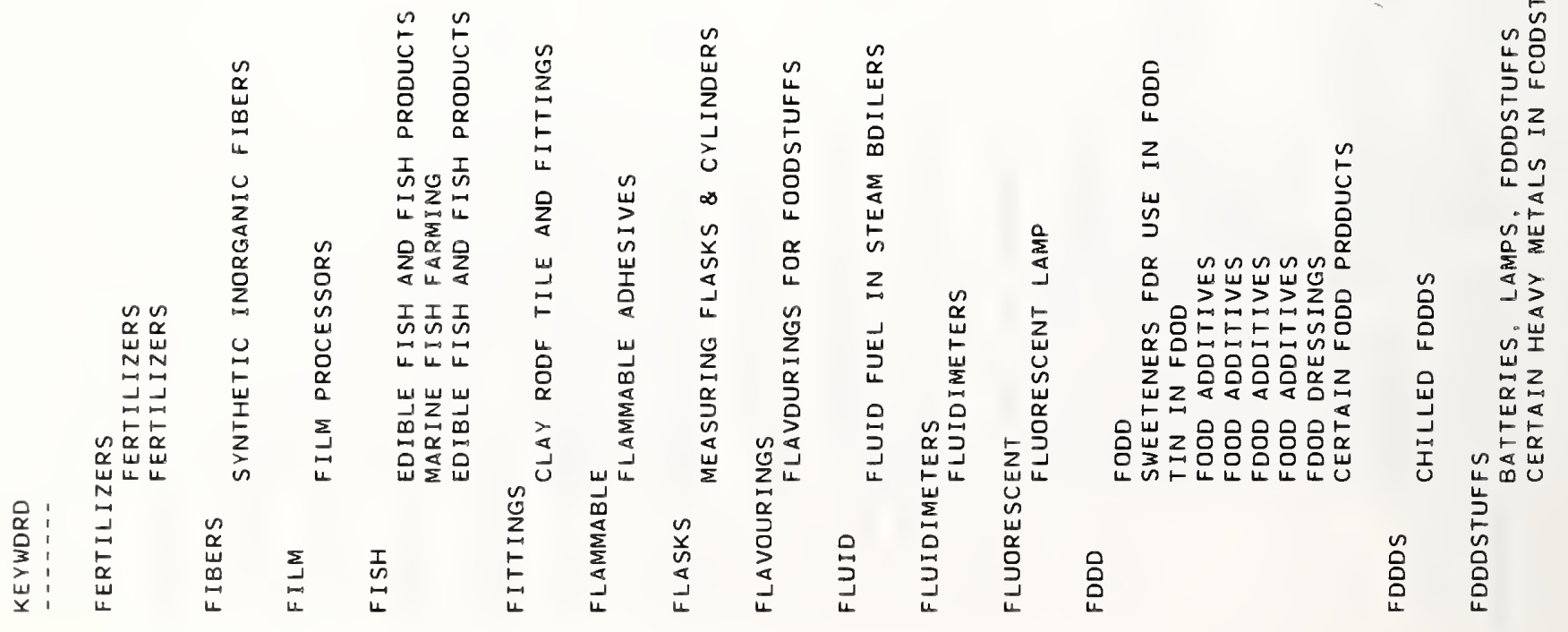




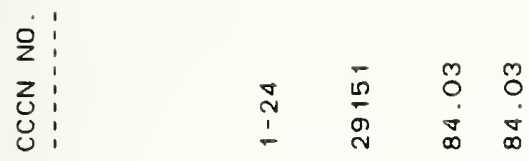

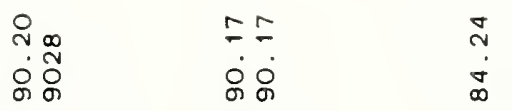

ஜั

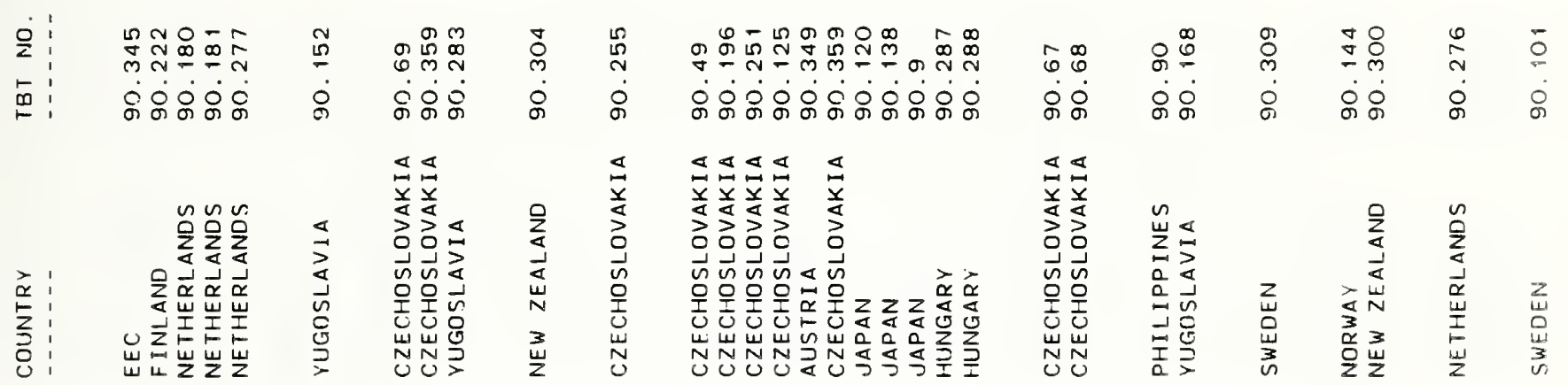

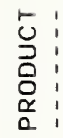

告

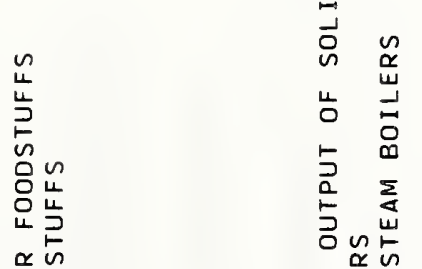

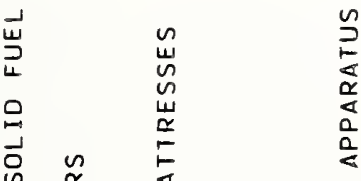

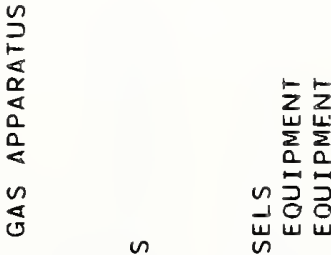

舟

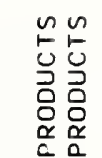

운은

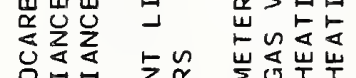

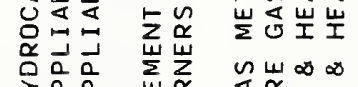

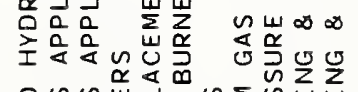

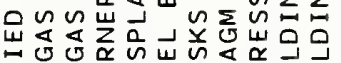

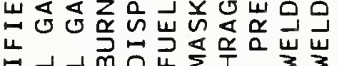

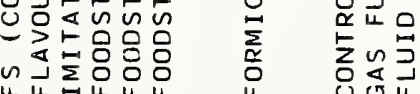

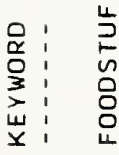


:

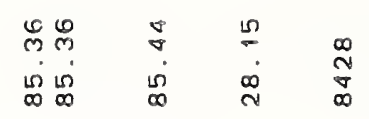

$\stackrel{\text { 온 }}{2}$

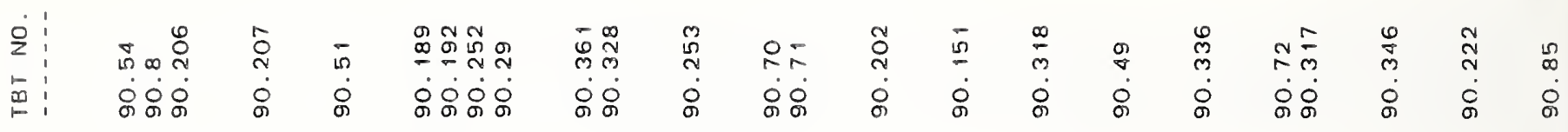

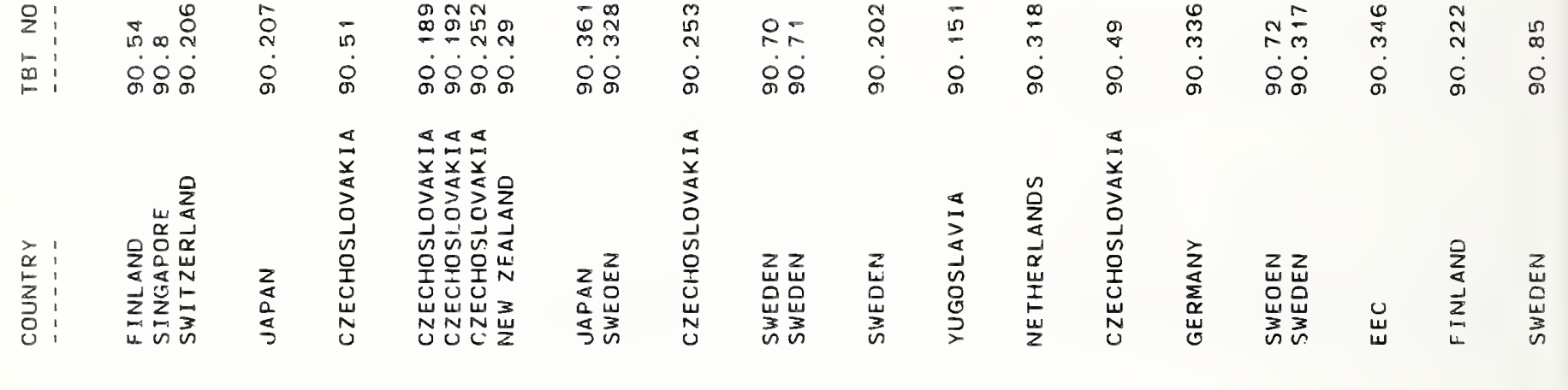

:
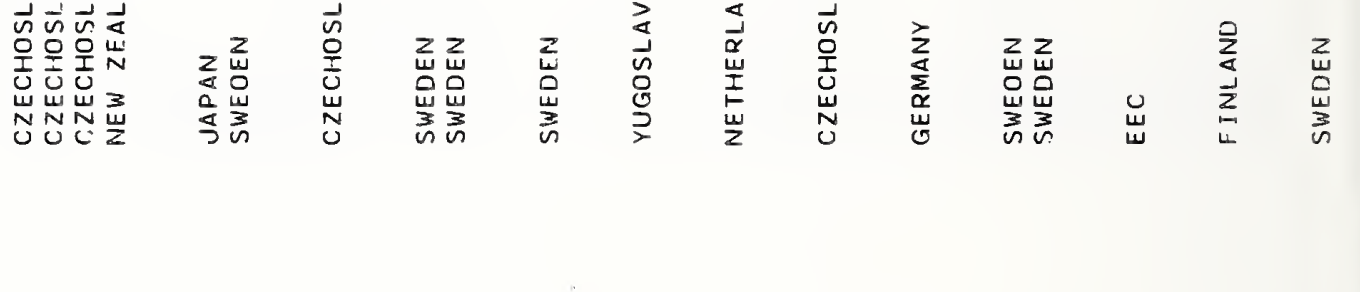

-

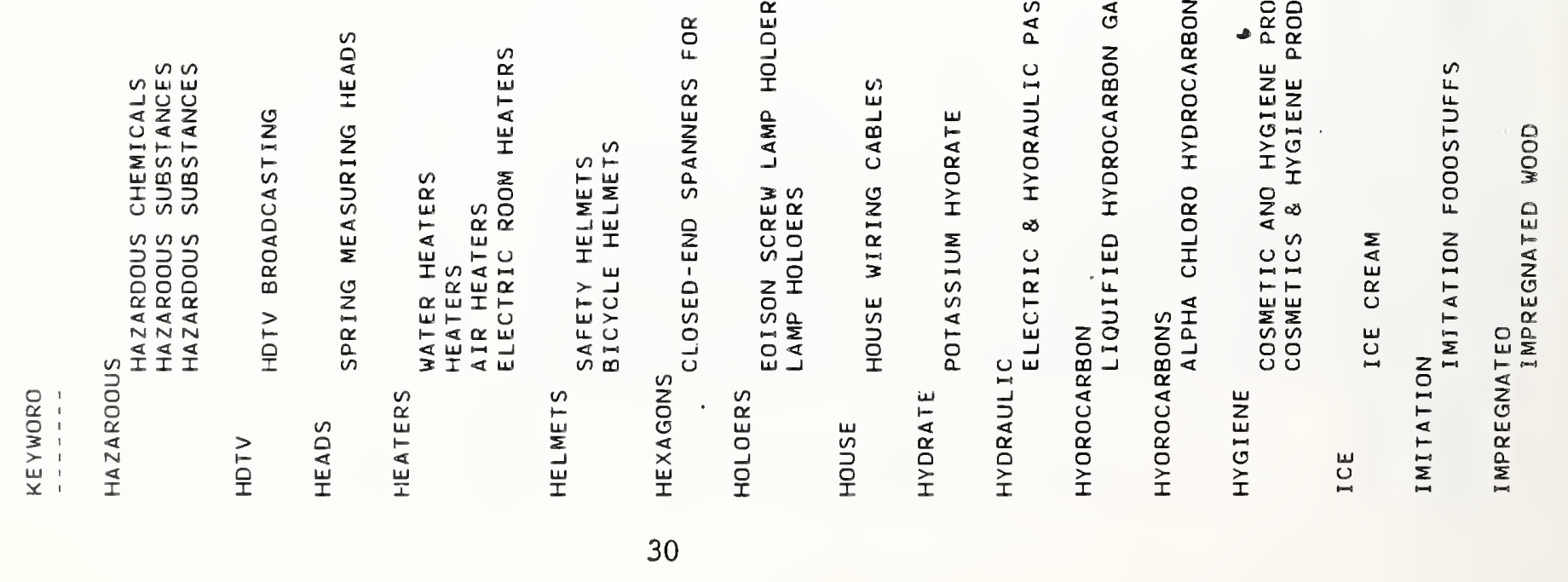




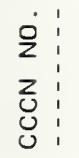

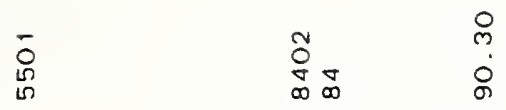

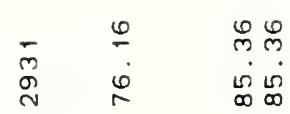

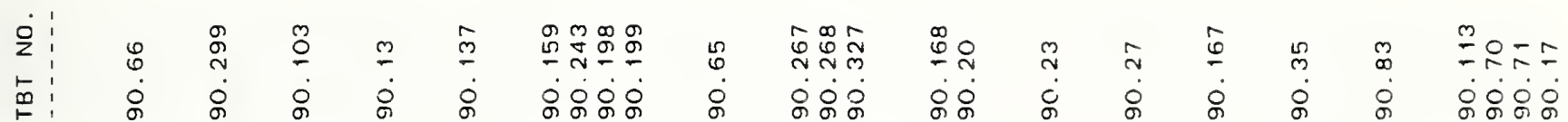

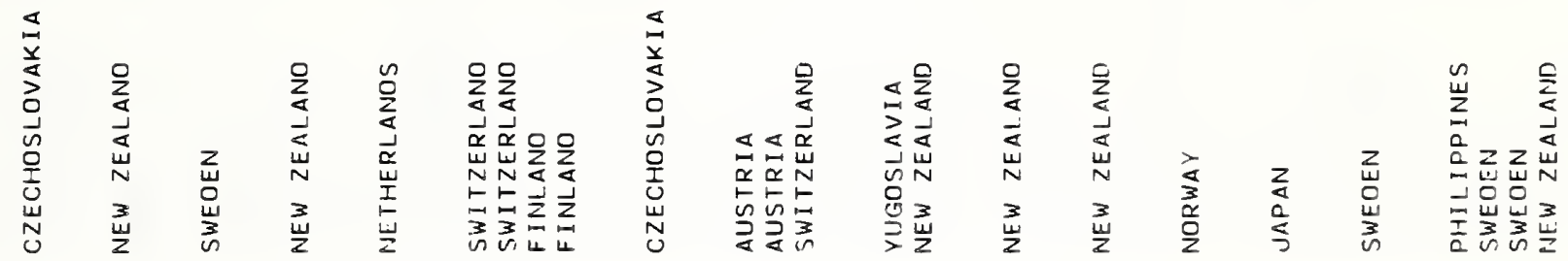

:

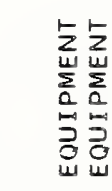




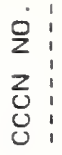

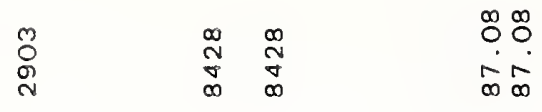

$\frac{\circ}{\stackrel{9}{\infty}}$

$\stackrel{i}{\dot{q}}$

แ)

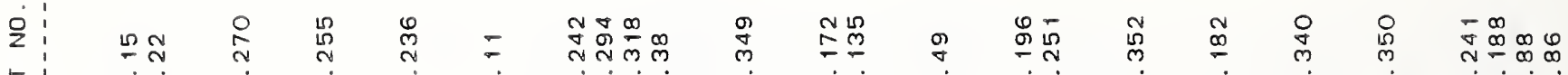
ட

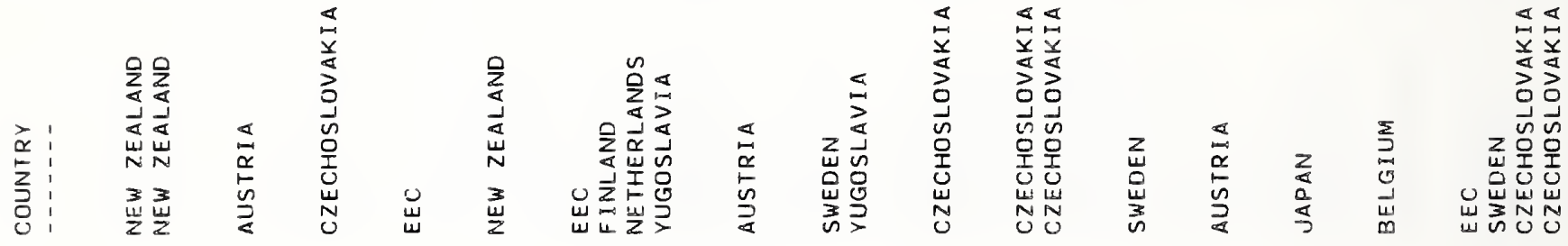

0
0
0
0
0
$\alpha$
$\alpha$
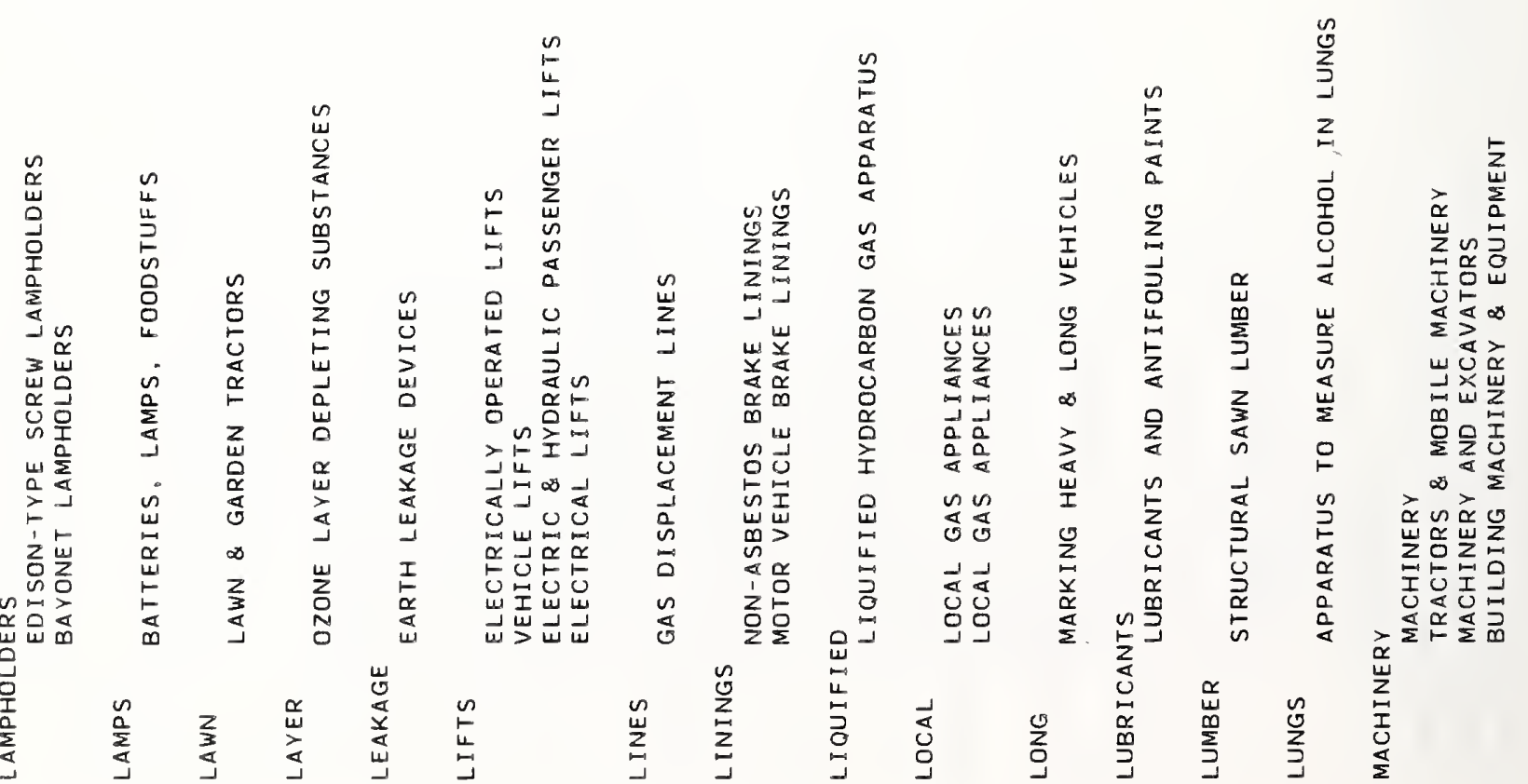


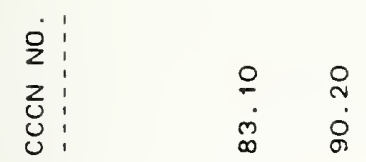

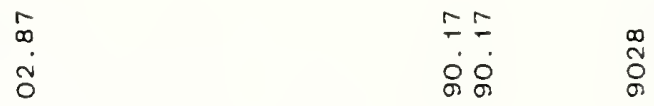

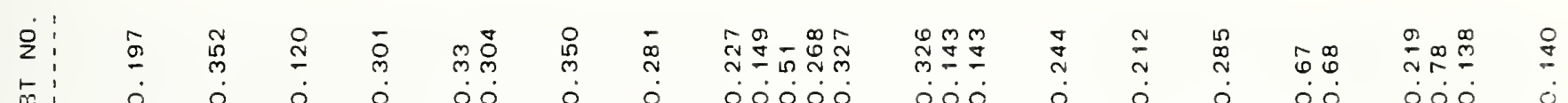

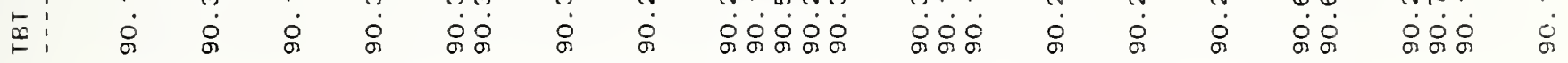

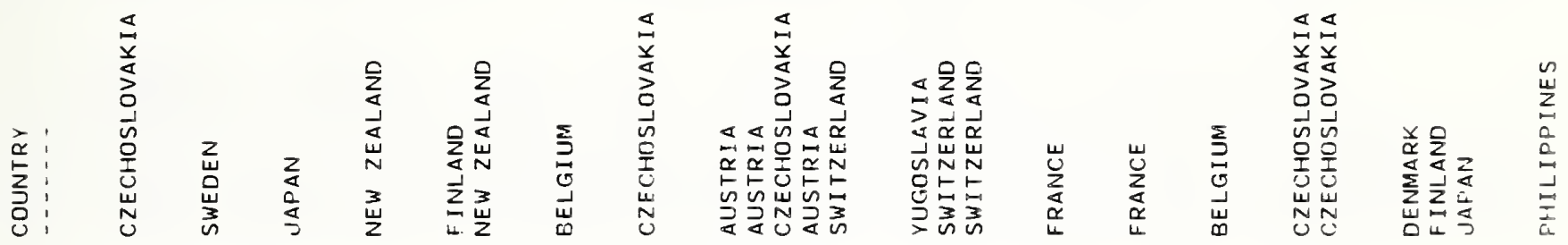

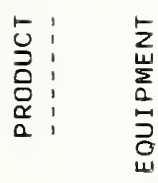

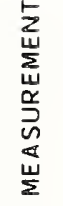

.

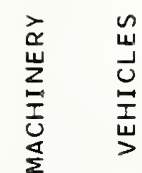

옹 


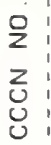

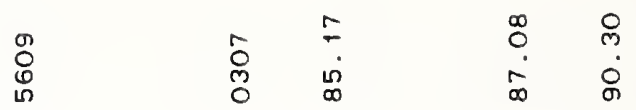

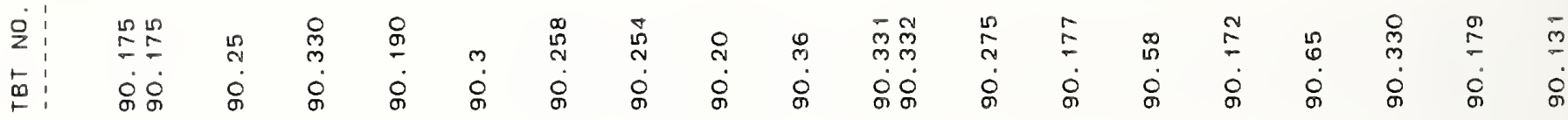

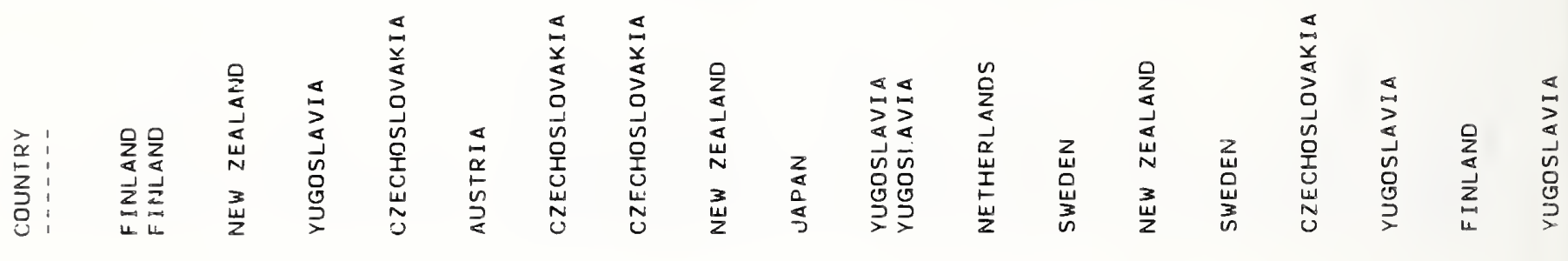

产:

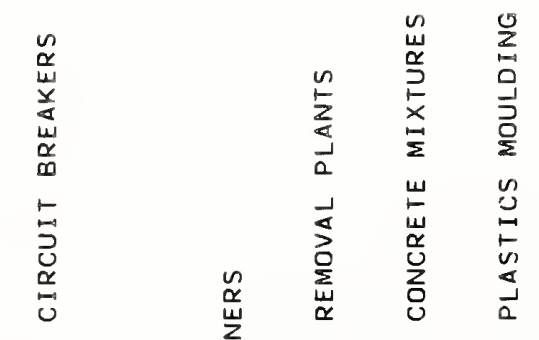

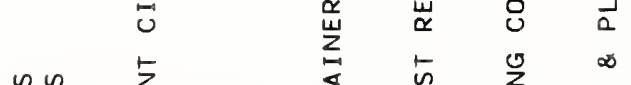

岁岕

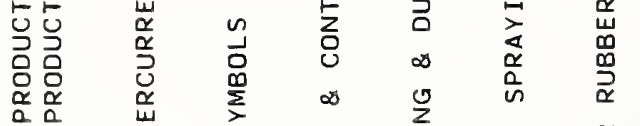




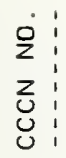

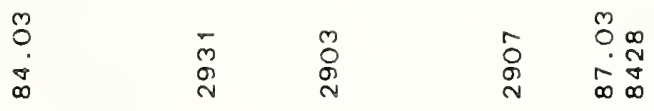

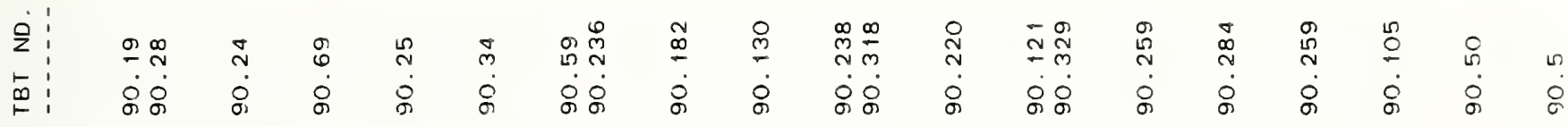

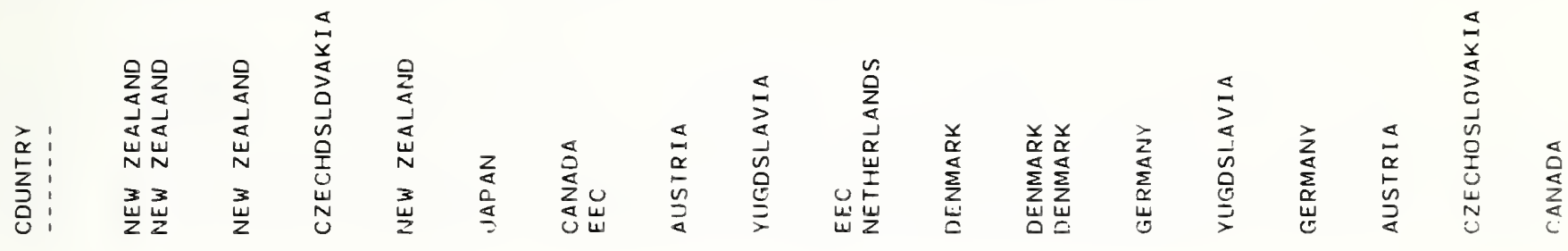

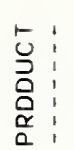

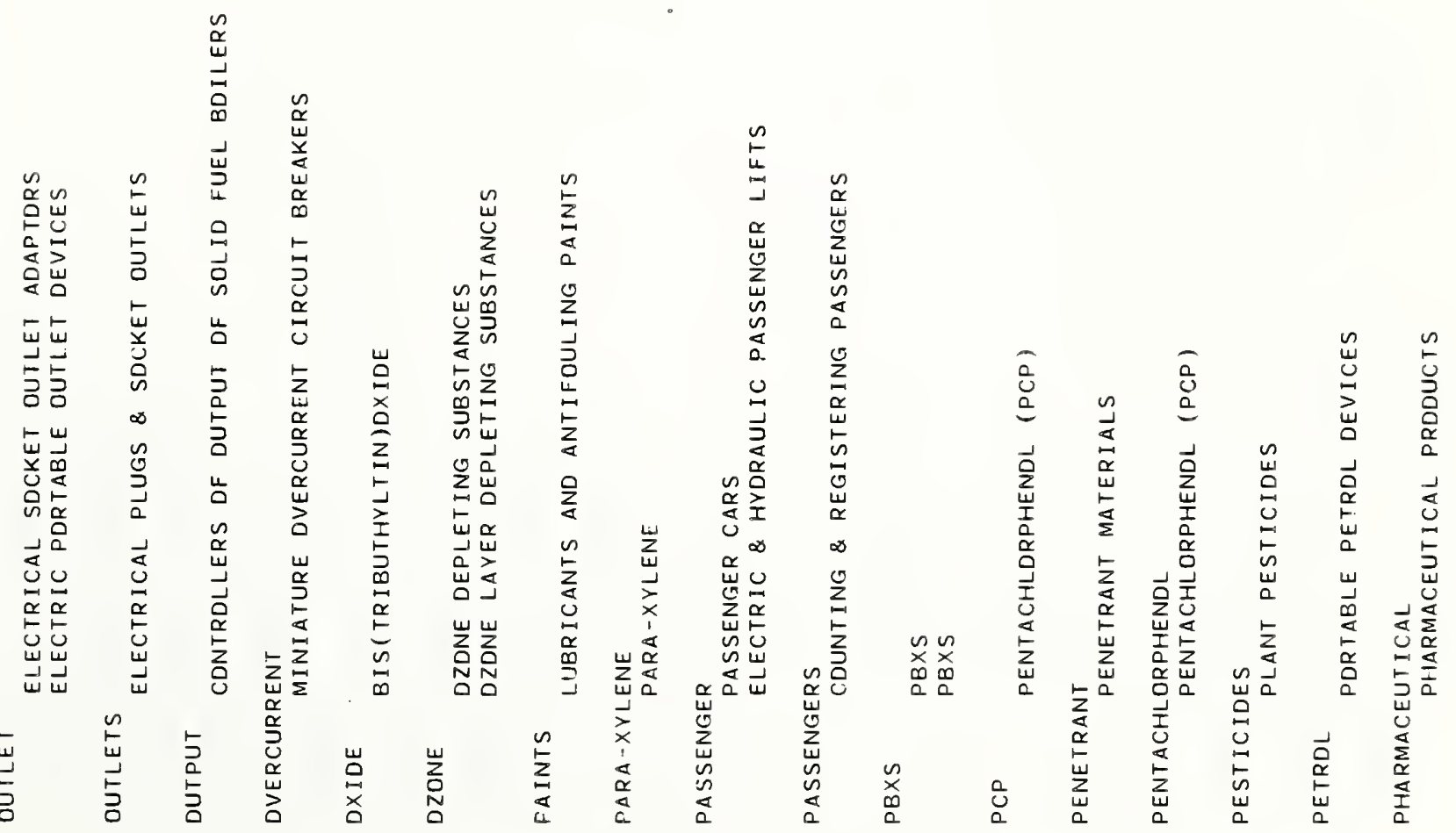




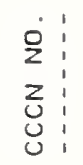

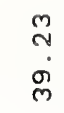

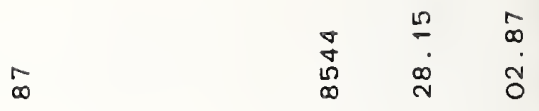

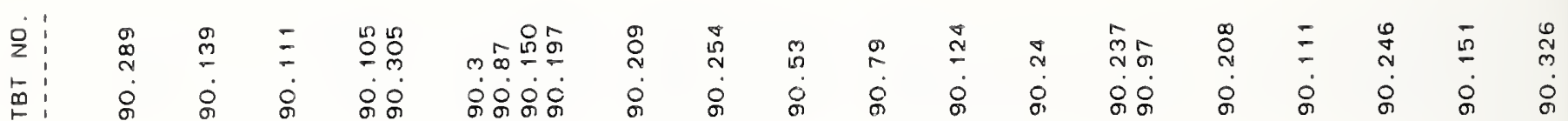

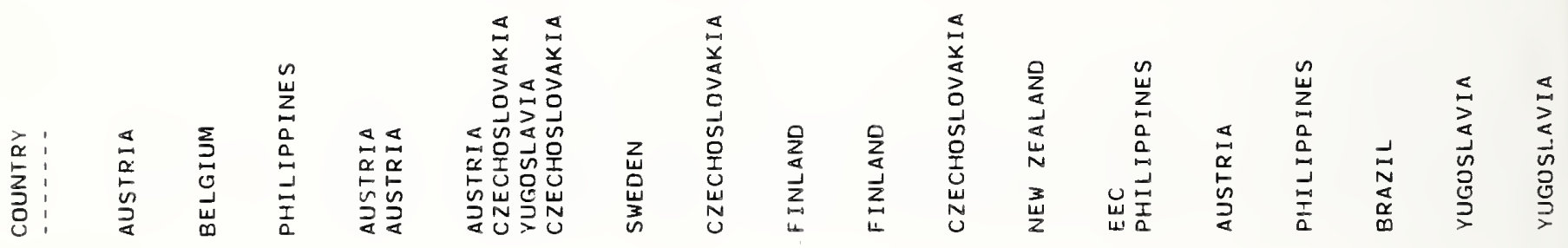

:

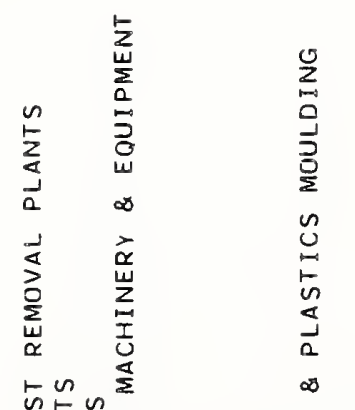

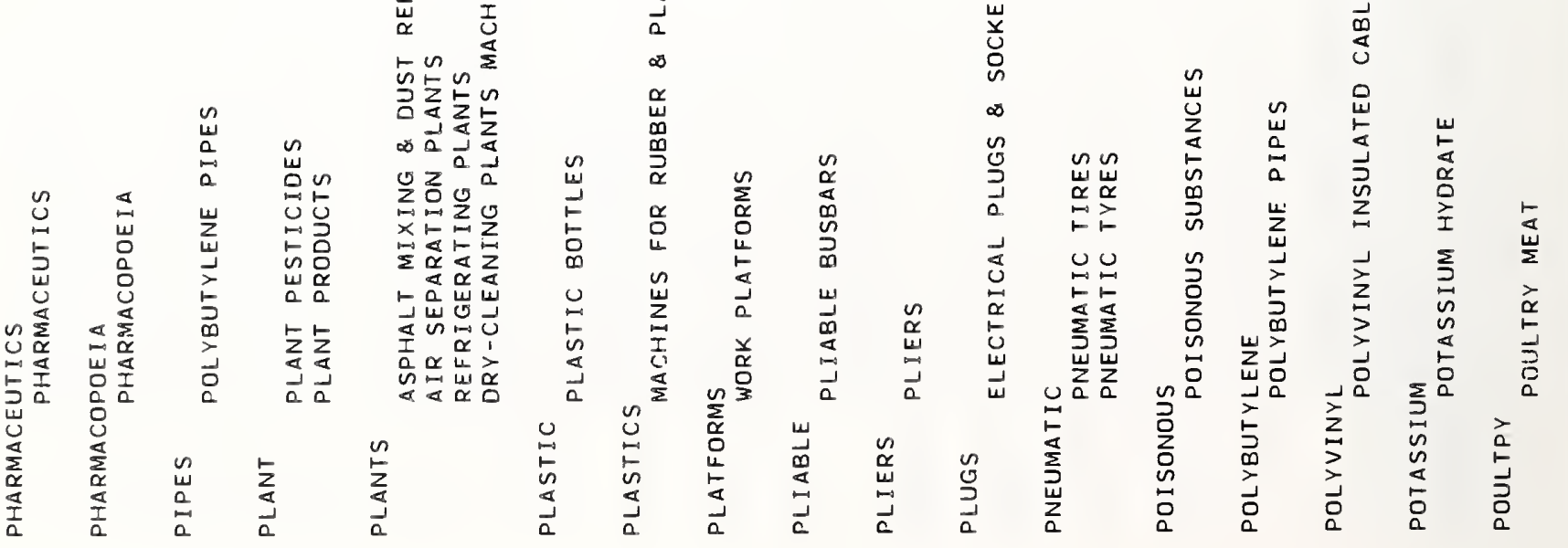


i

z:

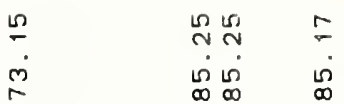

$\stackrel{10}{\infty}$

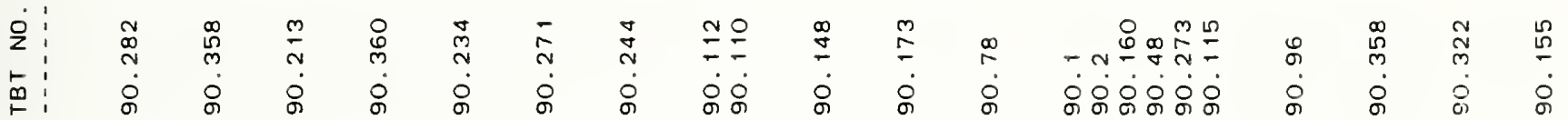

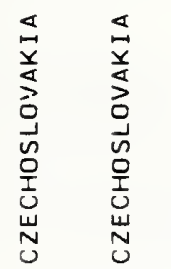

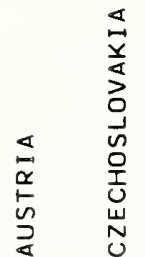

岂岂

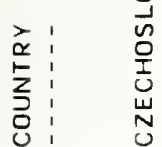

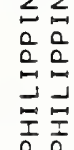

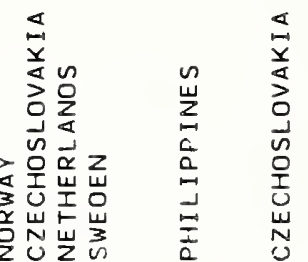
$\sum_{\frac{⿱}{\alpha}}^{\frac{\Sigma}{J}}$

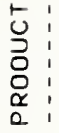

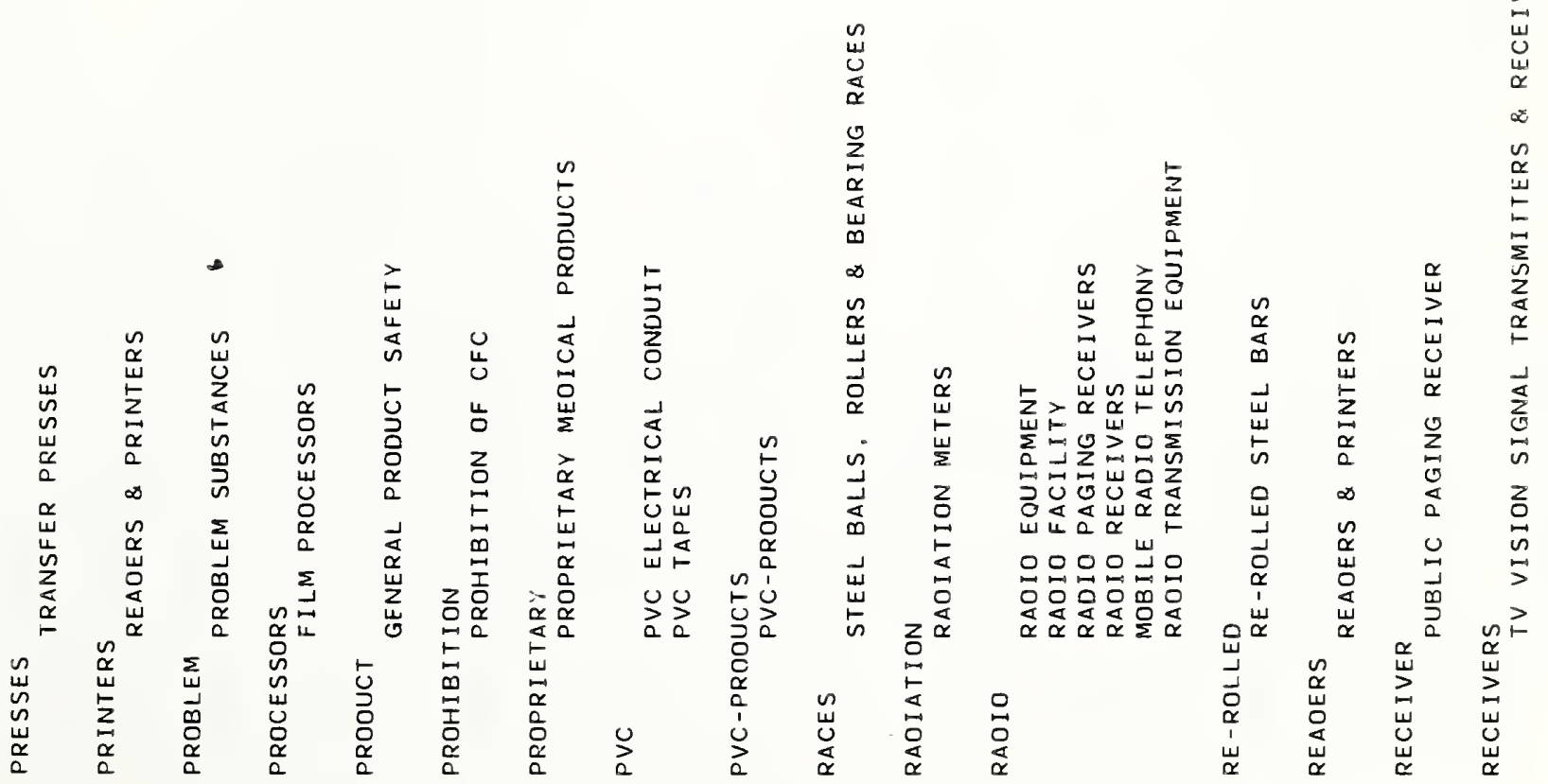


:

$\stackrel{n}{\stackrel{n}{n}}$

용

$\stackrel{\stackrel{\llcorner}{\sim}}{\stackrel{\infty}{\infty}}$

$\underset{\infty}{\infty}$

¿

ட

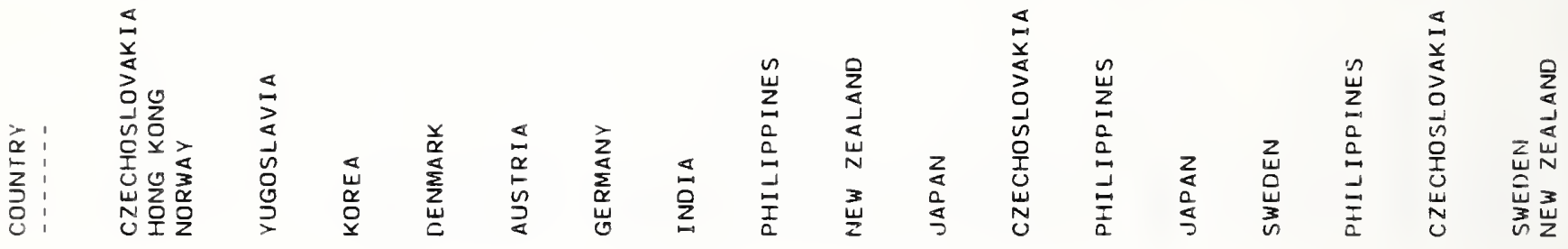

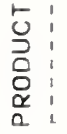

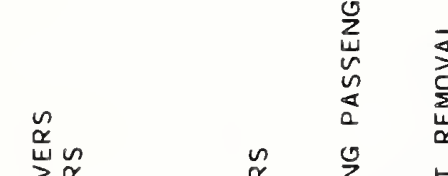




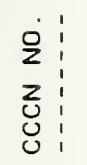

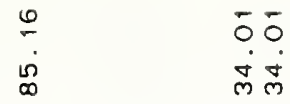

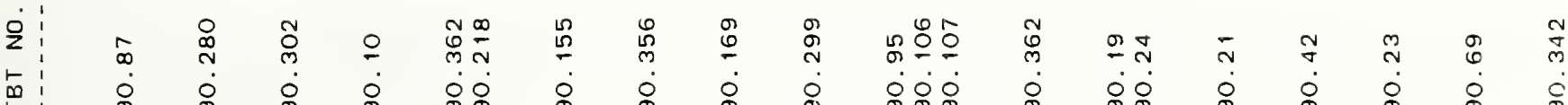

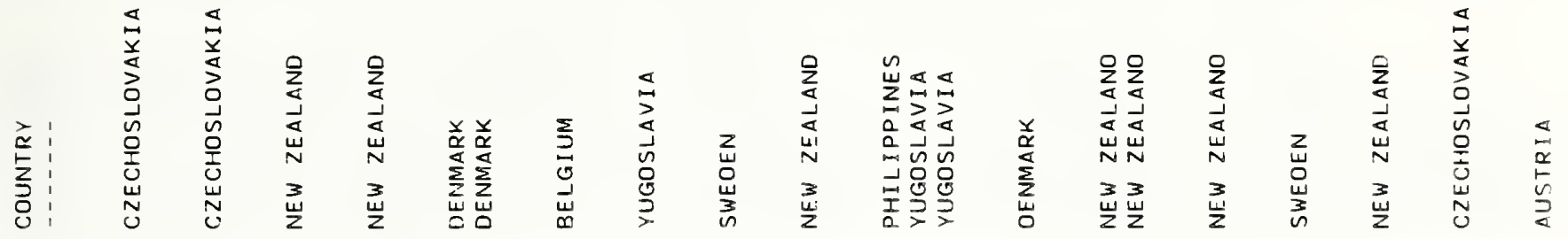

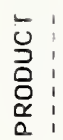




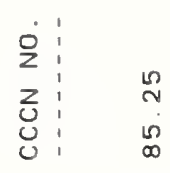

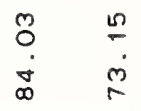

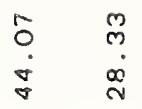

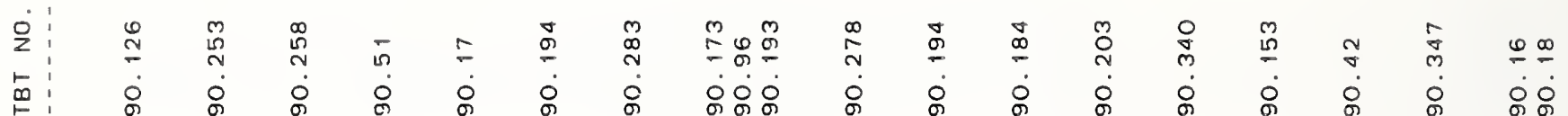

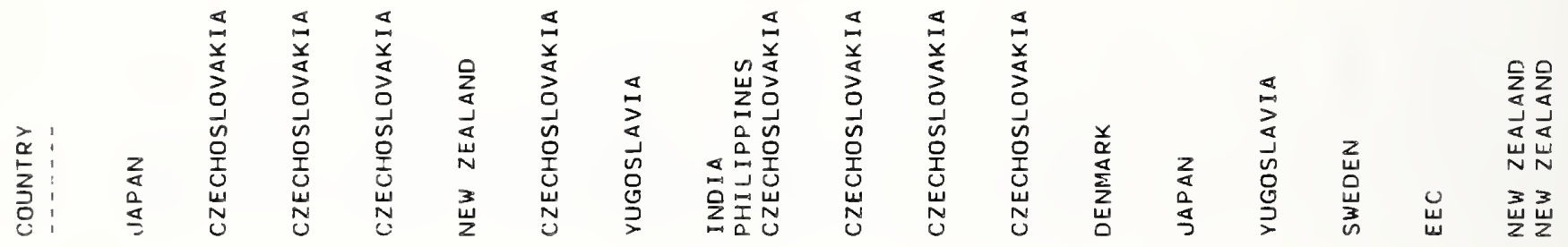

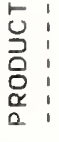

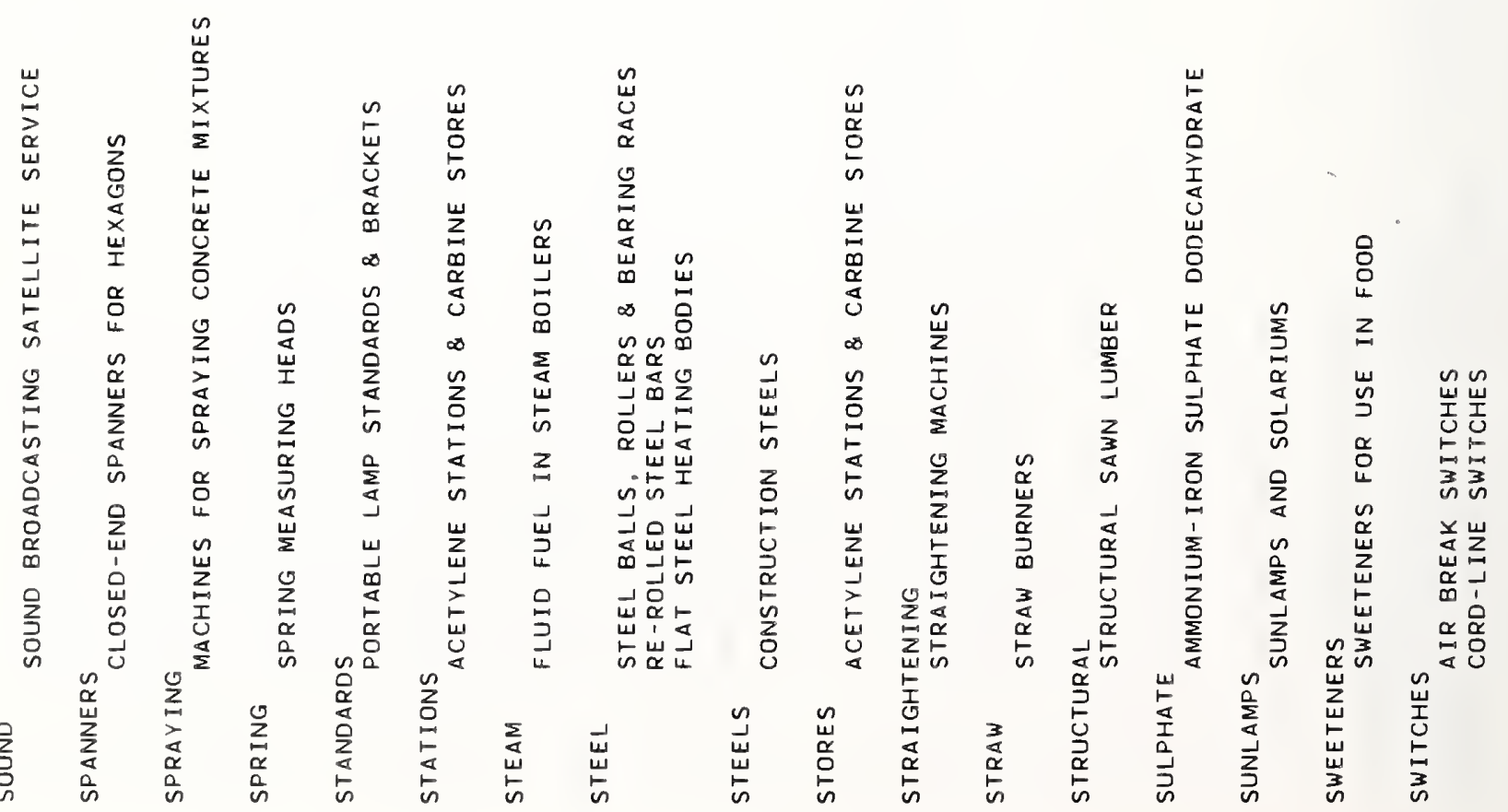


:

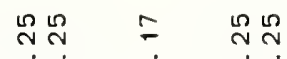

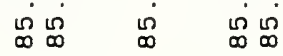

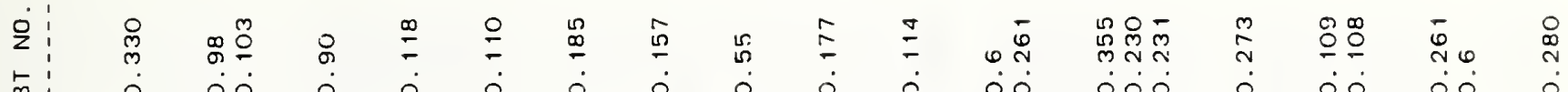
ஊ

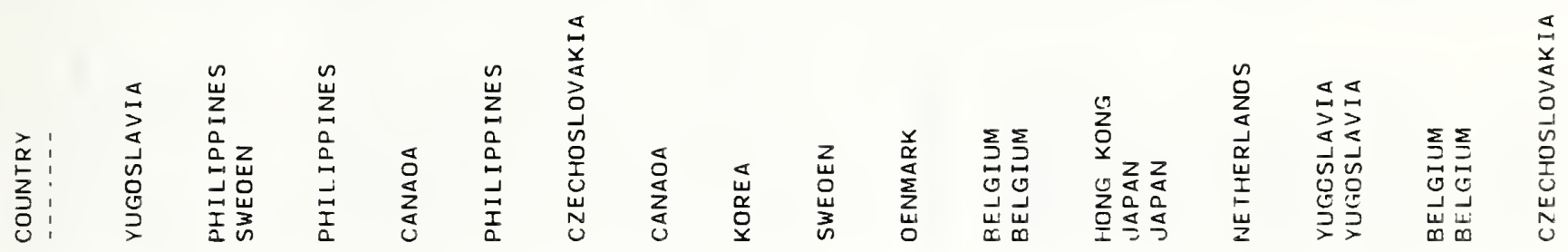

列
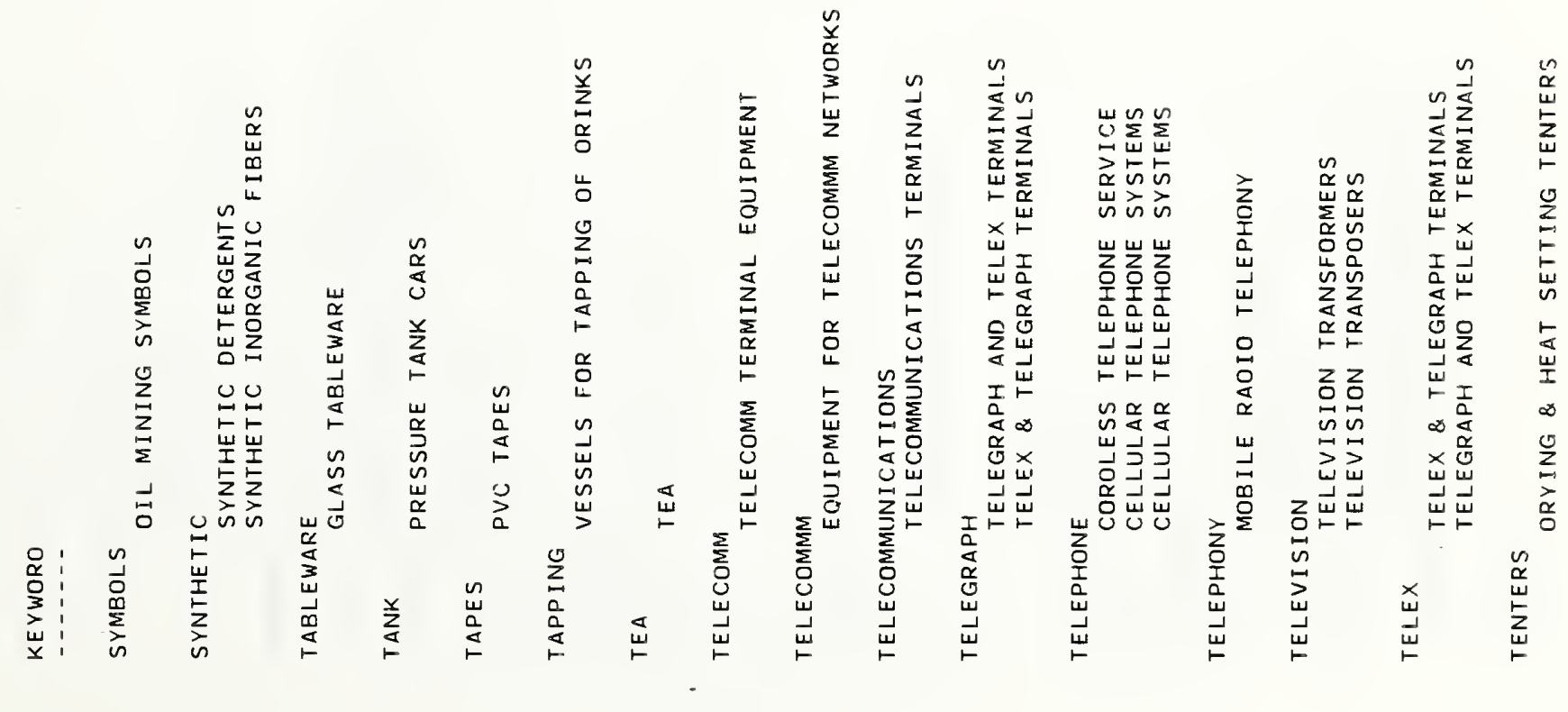
$\stackrel{2}{2}$

s:

ธิธีธิธ

लिख्ष

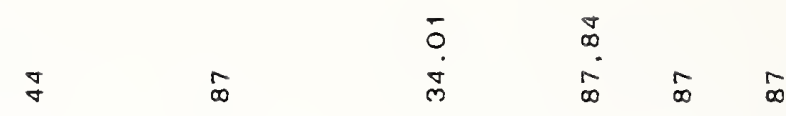

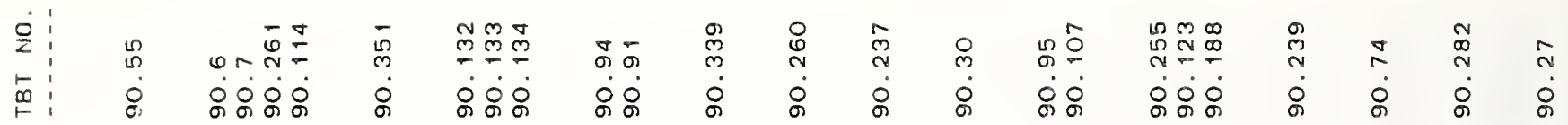

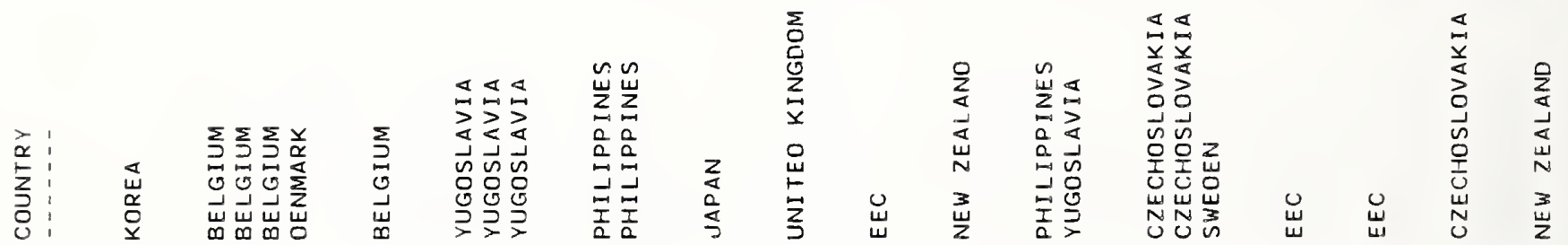

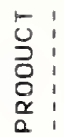
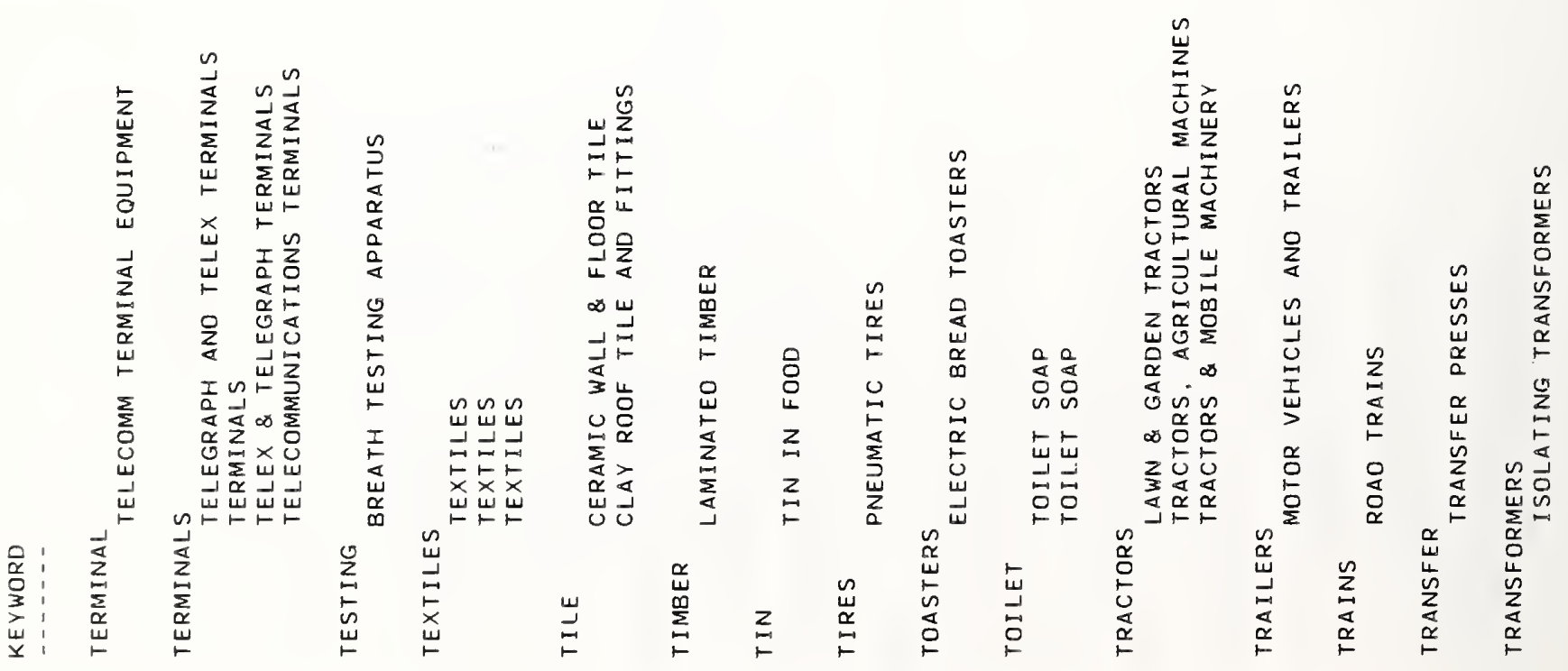
$\dot{2}$

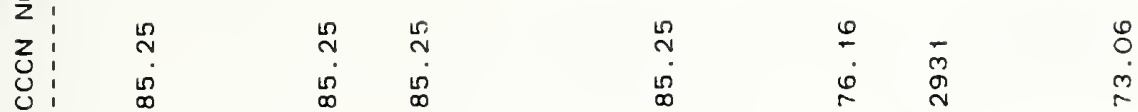

之) 年

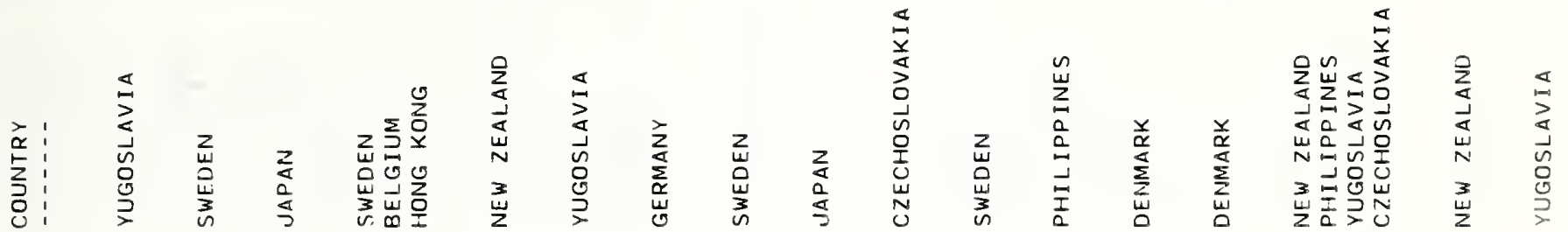

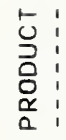

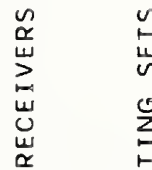




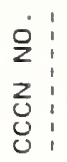

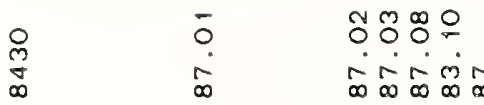

$\stackrel{0}{\infty}$

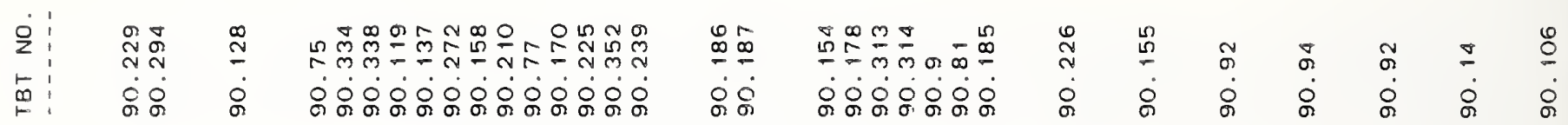

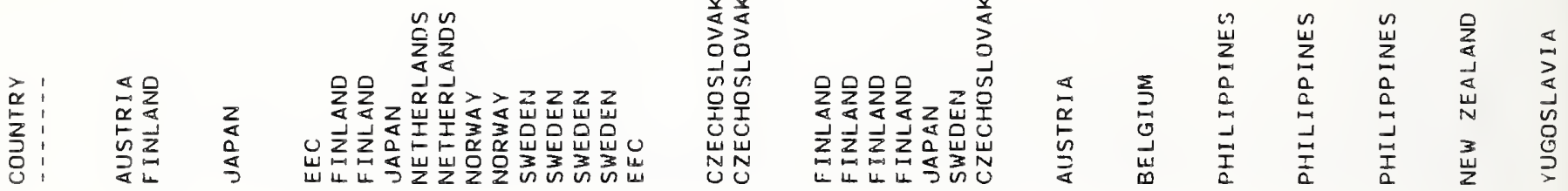

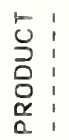

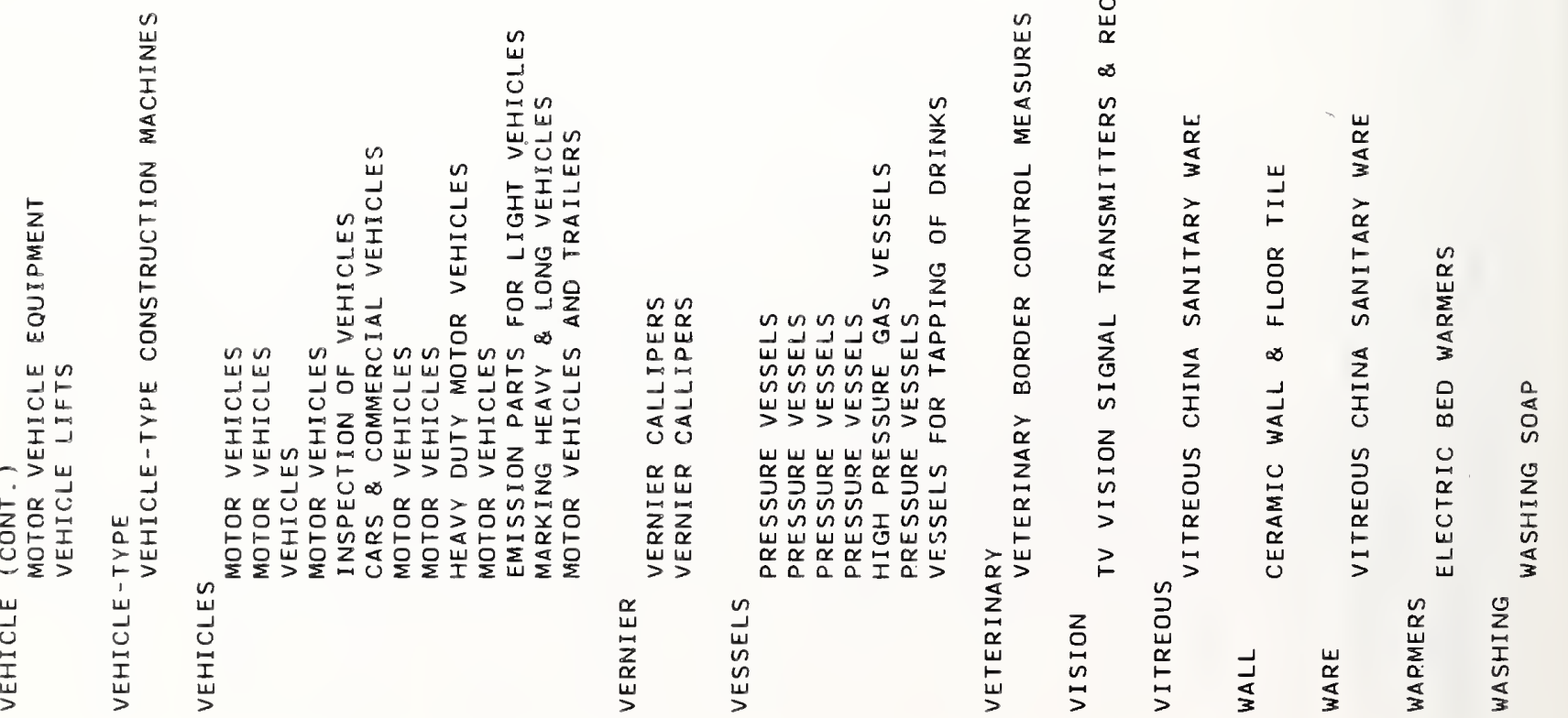


$\begin{array}{l:c}0 & 0 \\ z & 0 \\ 0 & 0\end{array}$

\begin{tabular}{lll}
$\stackrel{0}{0}$ & $\nabla$ & 0 \\
\hdashline & $\dot{0}$ & $\dot{0}$ \\
$\infty$ & $\infty$ & $\dot{\sigma}$
\end{tabular}

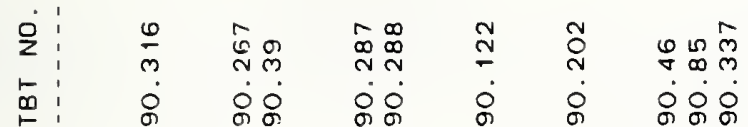

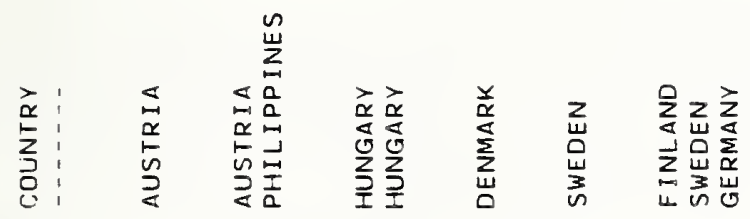

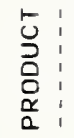

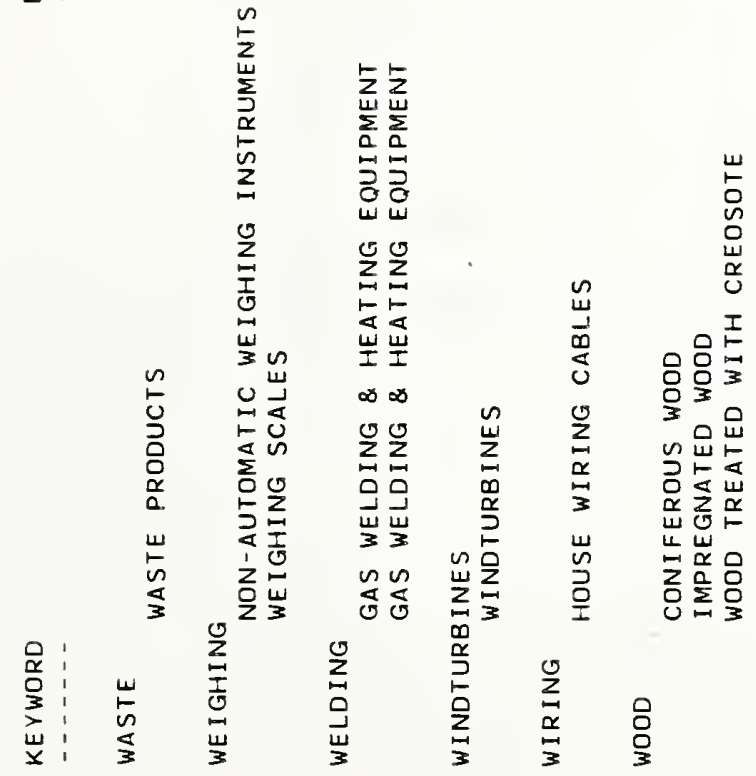



Party

Receiving

Enquiries

United

states

\section{Enquiries \\ originating In}

Argentina

Austria

Belgium

Brazil

Canada

Czechoslovakia

Denmark

Egypt

Finland

France

Germany

Greece

Hong Kong

Hungary

India

Ireland

Israel

Italy

Japan

Netherlands

New Zealand

Norway

Pakistan

Philippines

Portugal

Singapore

Spain

Sweden

Switzerland

Tunisia

United Kingdom

Yugoslavia

\section{NUMBER OF ENQUIRIES IN 1990}

\begin{tabular}{|c|c|c|c|c|c|}
\hline \multicolumn{2}{|c|}{$\begin{array}{l}\text { Total } \\
\text { (of which } \\
\text { from respective) } \\
\text { Enquiry Points) }\end{array}$} & \multicolumn{2}{|c|}{$\begin{array}{l}\text { Regarding } \\
\text { Proposed } \\
\text { Rules and } \\
\text { Regulations }\end{array}$} & \multicolumn{2}{|c|}{$\begin{array}{l}\text { Regarding } \\
\text { Existing } \\
\text { Rules and } \\
\text { Regulations }\end{array}$} \\
\hline $\operatorname{Rec}{ }^{\prime} \mathrm{d}$ & Answ'd & Rec'd & Answ'd & $\operatorname{Rec}^{\prime} \mathrm{d}$ & Answ'd \\
\hline$\underline{213}$ & $\underline{213}$ & 103 & 103 & 110 & $\underline{110}$ \\
\hline 1 & 1 & & & 1 & 1 \\
\hline 2 & 2 & & & 2 & 2 \\
\hline $9(7)$ & $9(7)$ & 6 & 6 & 3 & 3 \\
\hline $\begin{array}{c}5 \\
14(2)\end{array}$ & $\begin{array}{c}5 \\
14(2)\end{array}$ & 2 & 2 & $\begin{array}{r}5 \\
12\end{array}$ & $\begin{array}{r}5 \\
12\end{array}$ \\
\hline $\begin{array}{r}14(2) \\
6(6)\end{array}$ & $\begin{array}{r}14(2) \\
6(6)\end{array}$ & $\frac{2}{6}$ & 6 & & \\
\hline $14(12)$ & $14(12)$ & 2 & 2 & 12 & 12 \\
\hline 1 & 1 & & & 1 & 1 \\
\hline $11(10)$ & $11(10)$ & 5 & 5 & 6 & 6 \\
\hline $14(12)$ & $14(12)$ & 12 & 12 & 2 & 2 \\
\hline $10(1)$ & $10(1)$ & 2 & 2 & 8 & 8 \\
\hline 1 & 1 & & & 1 & 1 \\
\hline $1(1)$ & $1(1)$ & 1 & 1 & & \\
\hline $5(4)$ & $5(4)$ & 4 & 4 & 1 & 1 \\
\hline $12(9)$ & $12(9)$ & 9 & 9 & 3 & 3 \\
\hline 3 & 3 & & & 3 & 3 \\
\hline $2(1)$ & $2(1)$ & & & 2. & 2 \\
\hline $5(2)$ & $5(2)$ & & & 5 & 5 \\
\hline $1(1)$ & $1(1)$ & & & 1 & 1 \\
\hline $7(2)$ & $7(2)$ & 2 & 2 & 5 & 5 \\
\hline 3 & 3 & & & 3 & 3 \\
\hline $1(1)$ & $1(1)$ & 1 & 1 & & \\
\hline 1 & 1 & & & 1 & 1 \\
\hline $6(5)$ & $6(5)$ & 5 & 5 & 1 & 1 \\
\hline $4(1)$ & $4(1)$ & & & 4 & 4 \\
\hline $4(2)$ & $4(2)$ & 2 & 2 & 2 & 2 \\
\hline $4(4)$ & $4(4)$ & 2 & 2 & 2 & 2 \\
\hline $19(16)$ & $19(16)$ & 16 & 16 & 3 & 3 \\
\hline $7(1)$ & $7(1)$ & 1 & 1 & 6 & 6 \\
\hline $6(6)$ & $6(6)$ & 4 & 4 & 2 & 2 \\
\hline 31 (19) & 31 (19) & 19 & 19 & 12 & 12 \\
\hline $3(2)$ & $3(2)$ & 2 & 2 & 1 & 1 \\
\hline
\end{tabular}




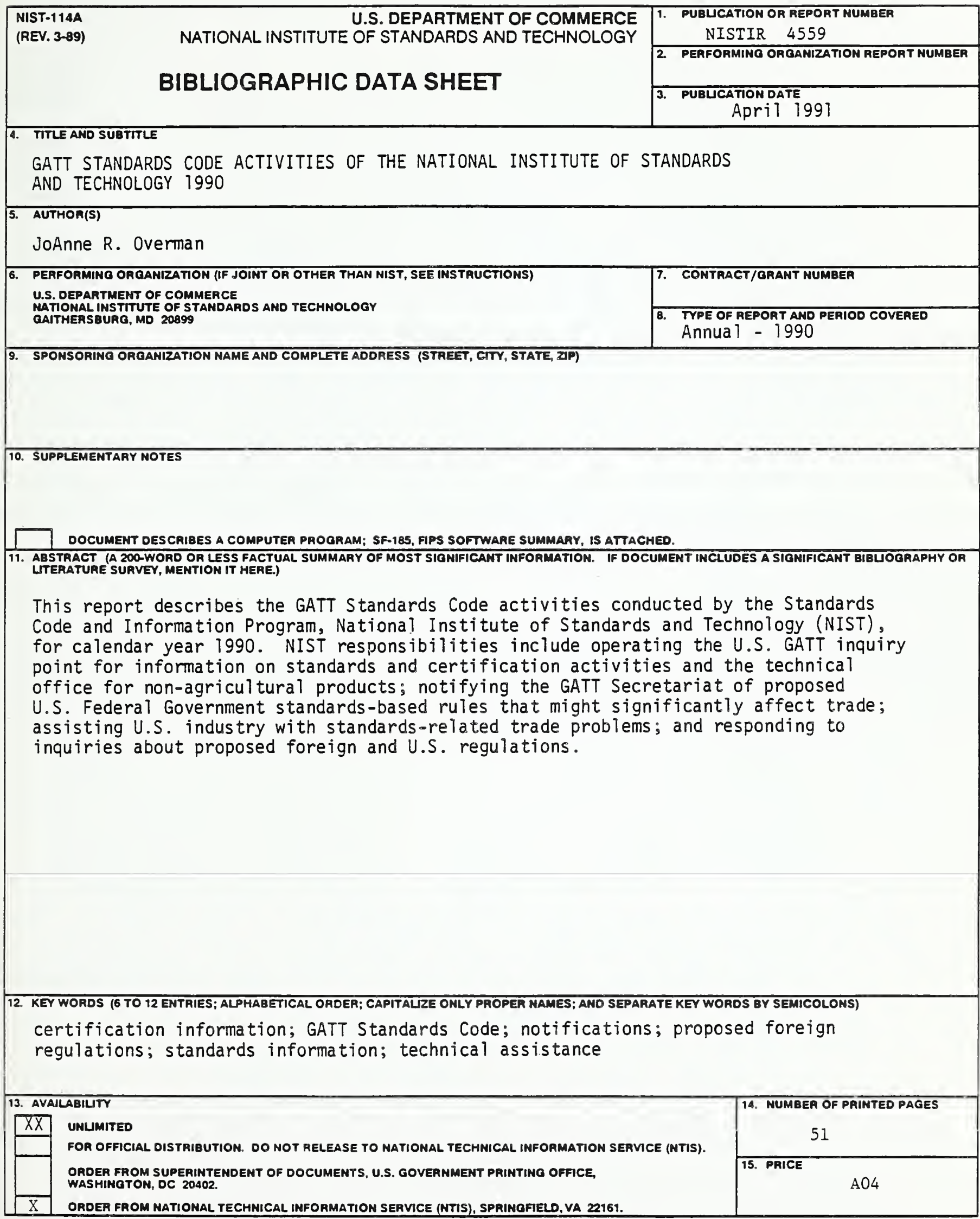

ELECTRONIC FORM 


